Imperial College

Business School

Does global drug innovation correspond to burden of disease? The neglected diseases in developed and developing countries

Eliana Barrenho, Marisa Miraldo, Peter C. Smith

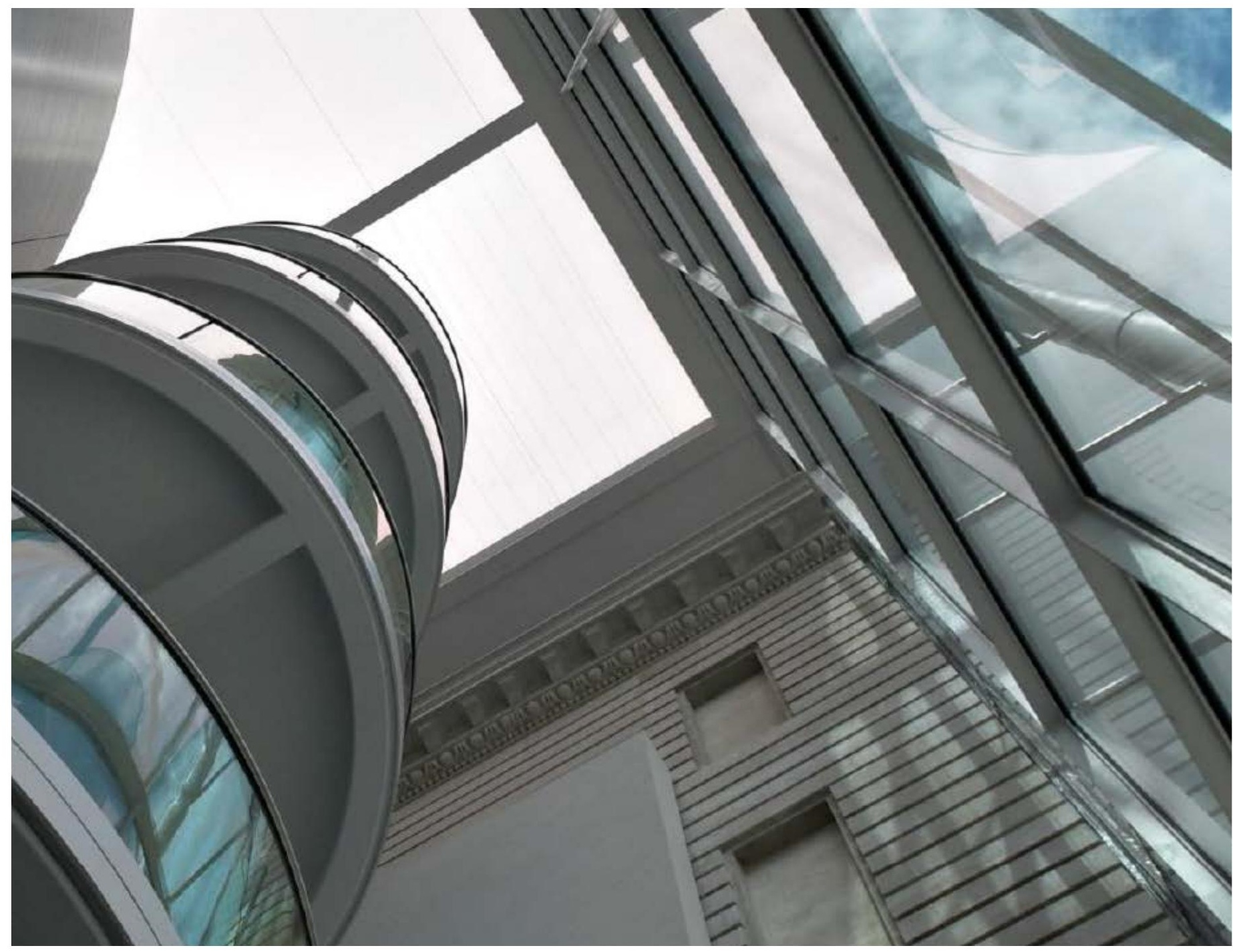

Discussion Paper 2017/07

October 2017 


\section{Does global drug innovation correspond to burden of disease? The neglected diseases in developed and developing countries.}

Eliana Barrenho, PhD*; Marisa Miraldo, PhD; Peter C. Smith, PhD.

Health Management Group, Imperial College Business School, Imperial College London, London SW7 2AZ, UK.

*Corresponding author: Eliana Barrenho. Details: email: eliana.barrenho@imperial.ac.uk, telephone: +44 (0)20 75941305 .

Keywords: Pharmaceutical Innovation, Inequalities, Burden of Disease, Market size, Concentration curves, Concentration indices. JEL codes: I140; I150; O32.

Manuscript word count: 5,745 words in main body (excluding references, tables and figures)

Manuscript table count: 7

Manuscript figure count: 8

Conflicts of interest: Authors have nothing to declare.

Funding sources: The authors acknowledge financial support available from Fundação para a Ciência e a Tecnologia, Ministério para a Educação e Ciência (FCT), Governo da República Portuguesa, Doctoral Fellowship POPH-QREN-SFRH-BD-69131-2010. 


\title{
Does global drug innovation correspond to burden of disease? The neglected diseases in developed and developing countries.
}

\begin{abstract}
While commonly argued that there is a mismatch between drug innovation and disease burden, there is little evidence on the magnitude and direction of such disparities. In this paper we measure inequality in innovation, by comparing R\&D activity with population health and GDP data across 493 therapeutic indications to globally measure: (i) drug innovation, (ii) disease burden, and (iii) market size.
\end{abstract}

We use concentration curves and indices to assess inequality at two levels: (i) broad disease groups; and (ii) disease subcategories for both 1990 and 2010.

For some of top burden disease subcategories (i.e. cardiovascular and circulatory diseases, neoplasms, and musculoskeletal disorders) innovation is disproportionately concentrated in diseases with high burden and larger market size, whereas for others (i.e. mental and behavioural disorders, neonatal disorders, and neglected tropical diseases) innovation is disproportionately concentrated in low burden diseases.

These inequalities persisted over time, suggesting inertia in pharmaceutical R\&D in tackling the global health challenges.

Our results highlight the priority disease areas for $R \& D$ investment in both developed and developing countries. 


\section{INTRODUCTION}

It is commonly argued that there is a misalignment between pharmaceutical innovation and global health needs, as indicated by the latest figures on global pharmaceutical research and development $(\mathrm{R} \& \mathrm{D})$ investments and the global burden of disease. Whether measured using the number of new chemical entities (NCEs) or the average sales per therapeutic area, R\&D investment has mainly targeted five therapeutic areas since 1990 (Pammolli, Magazzini, \& Riccaboni, 2011), namely (i) antineoplastics (53.2\% of total R\&D investment), (ii) antibacterials and antimycotics (18.4\%), (iii) treatments targeting the central nervous system (15.5\%), (iv) therapies for metabolism conditions (8.8\%), and (v) therapies to treat cardiovascular diseases (6.2\%).

These proportions correspond closely to pharmacological areas associated with disease prevalence in Western Europe, North America and Australasia (Murray et al., 2013) (namely, neoplasms, and mental and behavioral disorders (Murray et al., 2013)). However, the majority of the world's population lives in developing countries, for whom the disease burden is still mostly caused by infectious diseases and neonatal conditions (Murray et al., 2013), in stark contrast to the observed pattern of pharmaceutical innovation (Trouiller et al., 2002).

Although these figures hint at the apparent misalignment between global drug innovation and burden of disease (Table I), they cannot provide us with an accurate assessment of the nature and magnitude of that misalignment. Such an assessment requires a reconciliation of previously unrelated data sources to compare the distributions of innovation and burden of disease. This is the starting point of this study.

[Table I in here]

Two main reasons are generally put forward for the apparent mismatch. First, there are disease areas in which it is relatively more difficult to innovate. Therapies targeting these diseases are scientifically challenging and pose substantial safety issues. Second, firms act strategically when determining their R\&D investments by considering disease prevalence and incidence, and tend to concentrate on therapies targeting more profitable markets. There is therefore a lack of $R \& D$ activity targeting disease markets that are less attractive because of lower potential revenue from a new medicine, either because of low disease prevalence or low income levels (Drummond, Wilson, Kanavos, Ubel, \& Rovira, 2007; Dukes, 2002; Pecoul, Chirac, 
Trouiller, \& Pinel, 1999). The absence of incentive mechanisms promoting R\&D in riskier or less profitable disease areas may have contributed to a failure to deliver innovations in therapeutic areas with potentially large economic and health impacts (Kremer \& Glennerster, 2004; Scott Morton \& Kyle, 2011).

In order to inform policies to align R\&D activity better with societal needs it is essential first to identify and measure the nature of any mismatches between drug innovation and disease burden at a global level and examine how this has evolved over time. Hitherto only indirect evidence of the mismatch between drug availability and disease burden has been provided. A study in Spain suggests no correspondence between 477 economic evaluations performed between 1983 and 2008, and disease burden, whether measured as the years of lost life, years lived with disability, or disability-adjusted life years (DALYs) (Catalá-López et al., 2011). A second study suggests no correspondence between 512 published cost-utility analyses performed in the US between 1976 and 2001 and DALYs (Neumann et al., 2005). A third study finds a large concentration of a total of 2,381 clinical trials recorded in the World Health Organisation (WHO) International Clinical Trials Registry Platform relating to noncommunicable diseases (Viergever, Terry, \& Karam, 2013).

However the existing literature fails to measure directly the mismatch in drug innovation at a global level. In this study we therefore measure the dispersion of global innovation in terms of health, which we describe in detail in the methods section. We analyse the correspondence between drug innovation and disease burden measured by: i) DALYs, and ii) DALYs adjusted by ability-to-pay to construct a measure of market size.

\section{METHODS}

We perform four types of analysis. In the first we measure the correspondence between drug innovation and global disease burden and global market size across all diseases. In the second we carry out separate analysis for the two broadest disease groups as defined by the Global Burden of Disease (GBD) 2013, i.e. Communicable Diseases (CDs) and Non-communicable diseases (NCDs). In the third we refine the analysis by examining disease subcategories ${ }^{\mathrm{a}}$

\footnotetext{
${ }^{\mathrm{a}}$ Disease subcategories are the following: cardiovascular and circulatory diseases; Chronic respiratory diseases; Diabetes, urogenital, blood and endrocrine diseases; Diahrrea, lower respiratory infections, meningitis, and other common infections; Digestive diseases and Cirrhosis of the liver; HIV/AIDS and tuberculosis; Maternal disorders; Mental and behavioral disorders; Musculoskeletal disorders; Neglected tropical diseases and malaria; Neonatal disorders; Neoplasms; Neurological disorders; Nutritional deficiencies; Other communicable, maternal, neonatal, and nutritional disorders; and other noncommunicable diseases.
} 
belonging to the broad groups of CDs and NCDs causing the highest disability in developed and developing countries (using the country classification used by Institute of Health Metrics and Evaluation (IHME) in the GBD study (Murray et al., 2013)).

Finally, given its global public health relevance as shown by the GBD study (Murray et al., 2013), we also present the case of malaria and the neglected tropical diseases (NTDs) as defined by the Centre for Disease Control and Prevention (CDC) (Centre for Diseases Control and Prevention, 2011) and WHO (WHO, 2015) (Table II).

[Table II in here]

These analyses are performed for the years 1990 and 2010.

\section{Concentration curves and concentration indices}

We use concentration curves and concentration indices (Erreygers \& Van Ourti, 2011) using data from pharmaceutical innovation. Concentration curves allow assessing the degree of drug innovation inequality by plotting the cumulative percentage of drug innovation against the cumulative percentage of disease-specific burden of disease, ranked from the disease with lowest to the highest burden (horizontal axis). The 45-degree line represents a hypothetically equal distribution of drug innovation relative to disease burden. Curves lying above (under) the 45-degree line represent a concentration of drug innovation towards diseases with lower (greater) disease burden. We also undertake an analogous analysis substituting a measure of market size for the measure of disease burden.

We use the Erreygers index $E(r)$ (Erreygers, 2009) to calculate concentration indices and their standard errors to measure the magnitude and statistical significance of the inequality. It can be written as:

$E(r)=\frac{8}{n^{2}\left(b_{r}-a_{r}\right)} \sum_{i=1}^{n} \gamma_{i} r_{i}$

Where $\sum_{i=1}^{n} \gamma_{i} r_{i}$ denotes the sum of drug innovation $r_{i}$ targeting disease $i$ weighted the disease

ranked-dependent sum of disease burden and market size $\left(\gamma_{i}\right)$. The term $\frac{8}{n^{2}\left(b_{r}-a_{r}\right)}$ corrects for 
the boundness of the variable $r_{i}$, with $b_{r}$ and $a_{r}$, denoting respectively the maximum and minimum values of $r_{i}$, and $n$ the total number of diseases $i$ (Erreygers, 2009).

The sign of $E(r)$ indicates the direction of the inequality: positive values indicate a pro-high disease burden dispersion whilst negative values indicate a dispersion of drug innovation in favour of disease areas with low disease burden.

While there are other possible methodogical options to calculate concentration indices, such as the health concentration index introduced by Wagstaff et al. (1991), the normalized health concentration index by Wagstaff (2005), and the generalized health concentration index developed by Clarke (2002), these are not suitable given the nature of our data. The Erreygers' index $E$ satisfies the four desired properties crucial for this type of analysis, namely: (i) transferability, (ii) mirroring, (iii) level independence; and (iv) cardinal invariance (Van Doorslaer \& Van Ourti, 2011).

For the first property (transferability), $E$ ensures that, in the case of our study, transfers of drug innovation from higher (lower) to lower burden (higher burden) disease areas translate into pro-low burden of disease (pro- high burden of disease) changes in the inequality index. Second, the mirror property of $E$ ensures that a positive level of inequality is just the mirror image of the negative level of inequality (e.g. the level of inequality for health, measured by inequality indices, is just the mirror of the inequality indices for ill health and vice versa). This is true for $E$ even when the variables used for the construction of the index are bounded (i.e. variables that do not range from any negative to any positive value). This makes $E$ flexible enough to the point that the comparison across the sample of diseases/populations with different averages does not affect the calculation of the index. This is particularly relevant for our case since burden of disease and innovation are left- bounded variables.

Third, the level independence ensures that an equal increment of health for all individuals/innovation to all disease areas does not affect the index; that is, the index is invariant to scalar addition even when the bounds of the variable are kept constant.

Finally, the cardinal invariance of the index ensures that a linear transformation of our variables of interest (health variable/innovation) does not affect the value of the index; that is, the measured degree of inequalities is the same, irrespective of the cardinal scale of our variable. 
Data

In this study we combine three data sources: (i) IMS Health R\&D Focus database, which provides information on global pharmaceutical R\&D activity and market launches linkable through ICD-10 with (ii) the GBD 2013 study from Institute of Health Metrics and Evaluation (IHME) that includes the most recent global estimates on disease and country-specific disease burden and mortality for 1990 and 2010, and (iii) World Bank statistics on national GDP per capita to proxy ability-to-pay for 1990 and 2010.

The IMS database is used by the pharmaceutical industry to monitor market structure and performance to inform strategic decision-making. It assembles the history of all compounds in development from the early-1980s to the present, for which information is compiled from patent and regulatory filings, presentations at medical conferences, press releases, and financial information disclosed (IMS Health, 2011). It records the progress of compounds across R\&D phases, including discontinuation and market launch.

Compounds include small molecules, monoclonal antibodies, proteins, gene therapies, vaccines and immunotherapies, as well as fixed combination products, biosimilars, in vivo imaging agents, and specialized delivery systems targeting one of the 493 possible distinctive therapeutic indications (IMS Health, 2011). Each compound presents up to 17 different therapeutic indications. We have therefore considered all indications resulting in a compoundindication as our unit of analysis. These have been matched to disease-level need measures using ICD codes and medical dictionaries. After dropping compounds with missing information at the indication level ( $0.1 \%$ of total compound-indications) we have performed analysis for 1990 and 2010, with a total of 63,402 and 59,301 compound-indication units of observation respectively.

Using the three data sources, we construct three disease-specific variables for 1990 and 2010 to proxy: (i) global drug innovation, (ii) global burden of disease, and (iii) global market size.

To measure global drug innovation we use the number of successful compounds that effectively pass the drug approval and licensing requirements by counting the number of disease-specific therapies that have received market authorization in 1990 and 2010. 
While it could be argued that market launches fail to capture incremental innovations during the $R \& D$ process that never reach the marker, we believe that drug authorizations realistically capture the level of innovation effectively available to address population health needs affected by a specific disease. Innovations that do not successfully reach the market cannot be used for that purpose. For this reason we do not consider other potential measures of innovation such as the number of disease-specific patents, $R \& D$ investment, or disease-specific clinical trials.

We measure global disease burden using disease-specific DALYs as estimated in the 2013 GBD study. Despite the difficulties in measuring disease burden and health outcomes (Lopez \& Murray, 1998; Salomon et al., 2003), the 2013 GBD study is the main updated source of burden of disease data publicly available for 1990 and 2010 and provides consistent and comparable cause-specific estimates for mortality and DALYs for a list of 291 diseases across 187 countries (Murray et al., 2013). Therefore, we are able to match the ICD-10 codes for each cause category and subcategory in the GBD study to a cluster of therapeutic indications targeted by the compound projects in the IMS R\&D Focus dataset (IMS Health, 2011).

Market size for disease $i, \bar{m}_{i t}$, is constructed for 1990 and 2010 by summing up across all countries that suffer from that disease, a combined measure of disease burden associated with disease $i$ and ability-to-pay of the country in year $t$. We use World Bank statistics (World Bank, 2017) on national Gross Domestic Product (GDP) per capita (measured in purchasing power parity terms at 2005 constant \$US) to generate a global measure of ability to pay at disease level that is expressed as:

$\bar{m}_{i t}=\sum_{j}\left(\right.$ need $\left._{i j, t} \times G D P_{j, t}\right), \quad$ and $t=1990,2010$

where need $d_{i j, t}$ denotes the DALYs at time $t$ associated with disease $i$ for country $j$, and $G D P_{j, t}$ the GDP per capita for country $j$ in year $t$ (i.e. for both years of data 1990 and 2010).

We have excluded from the analysis the disease category "nutritional deficiencies" since the number of observations is insufficient to perform the disease subcategory analysis.

\section{RESULTS}

Table III shows the descriptive statistics on global drug innovation for the two broad disease groups, CDs and NCDs, in both years 1990 and 2010. Table IV provides similar descriptive analysis for disease subcategories. There is large dispersion of innovation across broad disease 
groups and disease subcategories. Whilst CDs show a substantial increase in the number of market launches from 208 in 1990 to 1,263 in 2010; the increase is more marked for NCDs from 748 in 1990 to 15,927 in 2010 (Table IV). The average number of market launches for each one of the 44 diseases consisting of the broad CDs group raises from 4.73 in 1990 to more than sixfold - i.e. an average of 30.07 market launches for the 42 diseases in the CDs broad group in 2010 (with standard deviations (sd) of 14.61 and 105.85 for both years, respectively). Yet, average number of market launches across NCDs shows much larger variations rising from 7.06 ( $\mathrm{sd}=14.07$ ) to 153.14 (sd =347.74) between 1990 and 2010.

The increasing sd in both broad disease groups hint large heterogeneity on the levels of innovation across diseases, as suggested by the descriptive analysis performed at disease subcategories level (Table IV).

[Tables III and IV in here]

Comparisons of average market launches at disease level when looking at descriptive statistics at disease subcategory level also show striking differences: the average number of market launches varies from 0 to 24.33 in 1990; and from 0 to 362.67 in 2010 (Table IV). For instance, in 1990, while the disease subcategory "neoplasms" presents an average of 10.56 new therapies across the 27 different types of cancer, the three diseases belonging to the subcategory "musculoskeletal disorders" exhibit an average of 24.33 new drugs. In 2010, for example, the 14 diseases that compose the subcategory "diahrrea, lower respiratory infections, meningitis, and other common infectious diseases” (Diahrrea and LRI henceforth) exhbit an average of 16.5 new therapies launched in the market, whereas for the 12 diseases that compose the subcategory “diabetes, urogenital, blood and endocrine diseases” show an average of 143.42 new market launches.

Morerover, the widening differences in the sd across subcategories and within subcategories between 1990 and 2010 also show evidence of increasing heteregoneity in the levels of drug innovation between diseases (Table IV).

There are also large variations in the causes of death and disease burden across countries. For some regions, disease burden has been persistently concentrated in the same disease groups since the nineties. In developed regions, such as North America and Western Europe, NCDs have for many decades been the major causes of death and disability. Conditions such as 
ischemic heart disease (IHD), chronic obstructive pulmonary disease (COPD), and cerebrovascular diseases, major depressive disorder (MDD), and trachea, bronchus and lung (TBL) cancers have presented the largest need for health care and innovation (Murray et al., 2013) (Table V). In many developing countries, such as those in Sub-Saharan Africa, disease burden continues to be concentrated in tuberculosis, lower respiratory infections (LRI), HIV/AIDS, malaria and diarrhea (Murray et al., 2013) (Table V).

\section{[Table V in here]}

However, there are regions experiencing structural changes in the epidemiological profile in the last two decades. In Southeast/East Asia and Oceania, the importance of diseases such as LRI and diarrhea that were prevalent in 1990 was replaced by cerebrovascular diseases, IHD, and stroke as main causes of disease burden in 2010. In Latin America and Caribbean, IHD emerges as the second cause of disease burden and diabetes the fifth cause of death in 2010 . The epidemiological transition is perhaps more evident in North Africa and the Middle East, where IHD, MDD, Stroke and low back pain are the main causes of burden in 2010, contrasting with LRI and diarrhea in 1990 (Murray et al., 2013) (Table V).

We now present the results from the analysis performed when using concentration curves and concentration indices to evaluate the level of correspondence between global innovation and global burden of diseases.

\section{Concentration curves and concentration indices}

The two broad disease groups: NCDs and CDs

Figure 1 illustrates the cumulative share of disease-specific innovation associated with global disease burden for both years, when ranking diseases in terms of DALYs and market size, respectively. Results from the concentration curves and indices (Table VI) show that innovation tends to be distributed according to burden of disease but concentrated towards diseases with larger market size.

\section{[Table VI and Figure 1 in here]}

When analyzing the dispersion of innovation for CDs and NCDs separately, the concentration curves suggest a concentration of innovation towards the diseases with the highest disease 
burden in both years (Figure 2). That is, there appears to be relatively more innovation in 'high burden' disease areas than is strictly merited, given the disease burden associated with those diseases. For instance, in 1990, CDs with associated higher burden such as lower respiratory infections (accounting for 5.23\% of total DALYs in CDs in 1990) show disproportionately more innovation (i.e. 21 new therapies launched in the market), compared to relatively lower burden diseases such as, for instance, leprosy and encephalitis (accounting for $0.003 \%$ and $1.22 \%$ of total DALYs in CDs in 1990) for which there has been disproportionately less innovation (i.e. zero and three new therapies launched in the market, respectively). Similarly in 2010, for high burden diseases such as the diahrreal diseases and the group of other infectious diseases (accounting for 1.21\% and 2.56\% of total DALYs in CDs in 2010, respectively) there have been, respectively, 60 and 572 new therapies launched in the market; whilst there have been no market launches for echinococcosis and vitamin A deficiency (that account for $0.02 \%$ and $0.10 \%$ of total DALYs in CDs, respectively) for instance.

\section{[Figure 2 in here]}

Likewise, NCDs associated with higher disease burden in 1990 such as cerebrovascular diseases and cervical cancer (accounting for, respectively, 5.02\% and 0.52\% of total DALYs for NCDs in 1990) show disproportionately more innovation (i.e. 6 and 16 market launches, respectively) than lower burden diseases such as eating disorders and endometriosis (that receive zero and one new therapies; accounting for $0.12 \%$ and $0.03 \%$ of total DALYs for NCDs, respectively). In 2010, for instance testicular cancer (accounting for $0.02 \%$ of total DALYs for NCDs) shows comparably lower innovation levels (with 58 new therapies launched in the market) than higher burden diseases such as nephritis and non-Hodgkin lymphoma (that account for $0.23 \%$ and $0.44 \%$ of total DALYs for NCDs in 2010, with 190 and 240 new therapies launched in the market).

The results from the concentration curves analysis are confirmed by the positive concentration indices reported in Table VI, although the concentration index is only statistically different from zero $(p<0.05)$ for NCDs in $1990(p<0.05)$. For NCDs in 2010 as well as for CDs in both years, even though the estimated concentration index is positive, the distribution of innovation is not statistically different from the equality line, and therefore we cannot reject the hypothesis of innovation being (equally) distributed according to disease burden. 
When market size is used as the basis for ranking, the results are qualitatively the same as above. The curves show similar unequal concentration towards disease areas that exhibit 'high market size' for both CDs and NCDs (Figure 3). These results are confirmed by the positive concentration indices (Table VI) that confirm that such inequality is statistically significant for NCDs in $1990(\mathrm{p}<0.01)$ and in $2010(\mathrm{p}<0.05)$, as well as for CDs in $1990(\mathrm{p}<0.01)$.

\section{[Figure 3 in here]}

We have further performed Friedman tests (Friedman, 1940) to assess statistical differences in the ranking of the diseases in 1990 compared with 2010 for: burden of disease, innovation and market size for both CDs and NCDs for all countries (Table VII) and for the group of developed and developing countries ${ }^{\mathrm{b}}$ separately (Table VII). Namely, we assess the statistical differences between 1990 and 2010 of the ranking of the diseases (ranked according to burden of disease and market size), by considering the total disease burden and total ability-to-pay for those groups of countries separately. Tests performed for the ranking of innovation in 1990 and 2010 consider the innovation level for the specific group of countries.

\section{[Table VII in here]}

Results suggest that there are statistically significant differences between the disease rankings of the two years considered for both disease burden and market size $(p<0.01)$ and that the diseases with more innovation in 1990 are statistically different from those in 2010 with a clear shift towards NCDs. In 1990 the three top diseases in terms of innovation were other infectious diseases, other neoplasms, and endocrine nutritional, blood and immune disorders. In 2010, other neoplasms, endocrine nutritional, blood and immune disorders, and other mental and behavioral disorders.

In the subcategory analysis, for the group of NCDs the ranking of diseases according to innovation activity differs between 1990 and 2010 for both developed and developing countries $(\mathrm{p}<0.01)$. However, for the case of CDs while the rankings of 1990 and 2010 are statistically different for developed countries $(\mathrm{p}<0.005)$ we can not reject the null hypothesis of rank similarity for developing countries.

\footnotetext{
$\mathrm{b}$ The categorization of developed and developing countries follows the United Nations (United Nations, 2017) classification available at http://unstats.un.org/unsd/methods/m49/m49regin.htm\#developed.
} 
The four disease subcategories with highest burden

The degree of inequality in favour of high burden diseases is more pronounced within some of the disease subcategories than the broad disease groups (as shown by the magnitude and significance of the concentration indices presented in Table VI). For the top four diseases that affect developed countries, there is a statistically significant skewing of innovation towards diseases with the highest disease burden in three of the four disease subcategories, i.e. “cardiovascular and circulatory diseases”, “neoplasms”, and “musculoskeletal disorders” (Figure 4a, 4b, 4c; respectively). These results are confirmed by the positive and statistically significant $(\mathrm{p}<0.01)$ concentration indices (Table VI).

For instance, in 1990 in the subgroup of "cardiovascular and circulatory diseases", there have been 29 new therapies launched in the market for cerebrovascular diseases (that accounts for 22.39\% of total DALYs in “cardiovascular and circulatory diseases” in 1990) but only four new drugs for cardiomyopathy and myocarditis (with 3.8\% of DALYs). In 2010, ischemic heart disease (that accounts for $44 \%$ of total DALYs in "cardiovascular and circulatory diseases” in 2010) exhibits 187 new drugs whilst hypertensive heart diseases (that accounts for $5.19 \%$ of DALYs) shows four new drugs.

Market launches in the subcategory "neoplasms" show similar patterns: in 1990, for cervical cancer there were 16 new drugs as opposed to one new drug targeting liver cancer (with the burden of disease being, respectively, 3.76\% and 1.19\% of total DALYs for "neoplasms” in 1990); in 2010, for instance 299 new therapies targeted kidney and other urinary organ cancers (accounting for 1.95\% of DALYs for “neoplasms” in 2010) compared to only two new drugs treating Hodgkin's disease (accounting for $0.34 \%$ of total DALYs in “neoplasms”).

Finally,the subcategory of "musculoskeletal disorders" also shows similar patterns: in 1990, rheumatoid arthritis, which accounts for $2.86 \%$ of total DALYs for "musculoskeletal disorders" in that year, had 19 new drugs launched in the market as opposed to 46 new drugs targeting osteoarthritis (which accounts for $8.96 \%$ of total DALYs in this disease subcategory); in 2010, the 197 new drugs targeting rheumatoid arthritis (accounting for $2.86 \%$ of total DALYs for "musculoskeletal disorders" in 2010) contrast with the 533 new drugs targeting 
osteoarthritis (which accounts for $10.1 \%$ of total DALYs for the same disease subcategory in 2010).

The results for market size are qualitatively similar for the disease subcategories “cardiovascular and circulatory diseases" $(\mathrm{p}<0.01)$, and “neoplasms" $(\mathrm{p}<0.01)$ but nonsignificant for "musculoskeletal disorders" (Figure 5a, 5b and 5c). Indeed, a close inspection of the concentration curve highlights that for this subcategory the direction of the inequality changes over the distribution: up to a certain level in the distribution the innovation is concentrated towards diseases that exhibit larger market size and affordability (e.g. gout, rheumatoid arthritis, and osteoporosis), while in the second part of the distribution innovations tends to be concentrated towards diseases associated with lower market size and affordability (e.g. neck pain \& low back pain).

[Figures 4-5 in here]

For "mental and behavioral disorder"s results are somewhat different (Figure $4 \mathrm{~d}$ and $5 \mathrm{~d}$ ). The negative and significant indices (Table VI) show an unequal distribution concentrated on diseases with lower burden (except in 1990, where it is not significant) and smaller market size. Results show that in 1990 conditions that rank lower in terms of associated disease burden such as, childhood conduct disorders (accounting for 3.74\% of total DALYs for mental and behavioral disorders in 1990) and schizophrenia (7.76\%) exhibit disproportionately more innovation (i.e. nine new therapies each disease) than diseases that rank higher in terms of disease burden such as anxiety (14.6\%) and bipolar disorders (6.78\%) presenting, respectively, six and three new therapies launched in the market in 1990. For 2010 results are qualitatively similar in that disorders that rank low in terms of associated disease burden. Disorders associated with the abuse of cannabis, opioids, and alcohol exhibit more innovation (i.e. 87 targeting each of the three diseases, and percentage of total DALYs for the subcategory "mental and behavioral disorders" in 2010, respectively, 1.11\%, 4.94\% and 9.52\%) than conditions with larger associated burden, with dysthymia, anxiety and unipolar depressive disorders showing respectively $5.98 \%, 14.49 \%$, and $34.11 \%$ of total DALYs in this disease subcategory, and eight new therapies launched in the market in 2010.

The results are also qualitatively similar when looking at market size. In both years disease areas with smaller market size (e.g. substance use disorders and child behavioural disorders in 1990; and asperger, autism and disorders associated with opiods abuse in 2010) show relatively 
more innovation than those with larger markets (e.g. dysthymia, bipolar disorders, schizophrenia and anxiety disorders).

Differences in the magnitude of the indices between 1990 and 2010 suggest increasing inequalities in the distribution of innovation in all disease subcategories except for "cardiovascular and circulatory diseases" (Table VI). Results suggest that, over time, innovation became increasingly more concentrated in diseases that rank high in terms of disease burden for subcategories such "neoplasms" and “musculoskeletal disorders”, and increasingly more concentrated towards diseases that rank low in terms of disease burden for the subcategory of "mental and behavioral disorders" (Table VI). Similar results are found for market size (Table VI).

For the disease subcategories causing the highest burden in developing countries, the concentration curves suggest a significant skewing towards diseases with highest burden and larger market size in the "diarrheal diseases and LRI”, and "cardiovascular and circulatory diseases" (Figures 6a and 6c; Figures 7a and 7c respectively) albeit less markedly than for the case of the developed countries. Results from concentration indices (Table VI) suggest significant $(\mathrm{p}<0.01)$ concentration of innovation towards diseases with highest burden and larger market size within these disease subcategories (with the exception of "diarrheal diseases and LRI” in year 1990 where results are not statistically significant for burden of disease analysis).

However, in 2010, there is a concentration of innovation in "neonatal disorders" with lowest burden and smaller market size as suggested by the statistically significant $(\mathrm{p}<0.01)$ concentration $^{\mathrm{c}}$. The comparison of the 1990 and 2010 concentration indices seem to suggest a decrease, albeit small, in the disparities.

[Figures 6-7 in here]

To summarize, while our results confirm quantitatively a mismatch between disease burden and innovation, we show that the pattern and direction of the inequalities are disease

\footnotetext{
c Note that we have excluded nutritional deficiencies from the analysis given the absence of any pharmaceutical innovation activity in this area. Given the nature of these diseases, the lack of innovation may simply signal that pharmaceutical innovation is not the most appropriate strategy for addressing need in this area.
} 
subcategory specific. While for some disease subcategories our results show innovation to be concentrated towards diseases with higher burden and larger market size (i.e. “cardiovascular and circulatory diseases” and "neoplasms”), for “mental and behavioral disorders" and "neonatal disorders" there is a clear concentration of innovation in diseases with lower disease burden and smaller market size. These are disease subcategories ranking top in disease burden in both the developed world (i.e. "mental and behavioral disorders”) and developing world (i.e. “neonatal disorders”).

The particular case of NTDs and Malaria

For the "NTDs and Malaria" subcategory, our results indicate a lack of innovation targeting the highest burden NTDs that affect predominantly developing countries (Figure 8). The concentration indices (Table VI) show a significant unequal distribution of innovation towards diseases associated with relatively lower burden and market size in both years 1990 and 2010 $(p<0.01)^{\mathrm{d}}$. To exemplify, dengue is responsible for $0.75 \%$ of total DALYs in "NTDs and malaria” and exhibits 12 new drugs launched in the market in 1990, as opposed to the three new drugs targeting rabies that is associated with a much larger burden (which account for $3.39 \%$ of total DALYs in "NTDs and malaria” in that year). In 2010, malaria, responsible for 81.23\% of total DALYs in “NTDs and malaria”, exbihited nine new drugs launched in the market as opposed to the 21 new drugs targeting chagas disease, which accounts for a relatively smaller disease burden, i.e. 0.04\% of total DALYs in “NTDs and malaria” in 2010.

[Figure 8 in here]

\section{DISCUSSION}

We have assessed inequality in drug innovation considering all innovation activity across all therapeutic and disease areas for 1990 and 2010. Our results confirm quantitatively assertions about the mismatch between disease burden and pharmaceutical innovation. However, the direction of the inequalities varies across disease areas.

On the one hand, in both 1990 and 2010 drug innovation has disproportionately favoured diseases with higher burden and larger market size, for the "cardiovascular and circulatory

\footnotetext{
d We have performed the analysis for NTDs only excluding Malaria and while results for 2010 remain qualitatively the same, for 1990 the concentration indices are not significant suggesting that we can not reject the hypothesis of distribution of innovation according to disease burden and market size. Results available from the authors upon request.
} 
diseases", "neoplasms" and "musculoskeletal disorders" groups. These are diseases mostly prevalent in the developed countries where the number of patients and the willingness-to-pay of payors makes these markets finantially attractive to the pharmaceutical industry. Moreover, the increased burden of disease associated with these NCDs in developing countries as a result of the epidemiological convergence between developed and developing countries (Murray et al., 2013; Nathan, 2011) enlarges these markets, and consequently the prospects of profitability in these disease areas at a global level.

On the other hand, there is a substantial misalignment between disease burden and drug innovation for specific disease areas, ranking top causes of death and morbidity in both developed and developing countries. These are respectively “mental and behavioral disorders”, and "neonatal disorders". Drug innovation targeting top causes of burden in developed countries for diseases that are part of "mental and behavioural disorders" has been concentrated in disease areas that rank low in terms of associated disease burden. This result has persisted over time and our results suggest that inequality has been increasing. Likewise, innovation targeting "neonatal disorders" that mostly affect developing countries has been disproportionatly concentrated in disease areas with lower burden of disease.

Also, and following a similar pattern, innovation targeting "NTDs and Malaria” has been unequally concentrated in diseases that have a relatively low burden in the developing world. In fact, these inequalities have reduced between 1990 and $2010^{\mathrm{e}}$.

These results strengthen the case for intervention in global R\&D markets, in the form of better alignment between public and private sector R\&D strategy and health need (Fauci \& Morens, 2012; Nathan, 2011), and the urgent need to redesign public policies (Cech, 2005) to foster innovation in neglected disease areas in both developed and developing countries.

Finally, our results suggest some inertia in pharmaceutical industry $R \& D$ strategies with regards to developing innovation targeted at CDs prevalent in developing countries, over the last two decades. This implies that, in those countries, morbidity and mortality associated with those diseases will remain unadressed. Therefore, while those that suffer from NCDs will benefit from novel treatments, others will not have any innovation to address their needs. This

\footnotetext{
${ }^{\text {e }}$ However, when we perform analysis over time for the NTDs alone (i.e. excluding malaria from the analysis) inequalities have increased.
} 
may result in widening inequalities within developing countries (Marmot, 2005; Monteiro, Moura, Conde, \& Popkin, 2004).

It should be remarked that equal distribution of drug innovation, in line with disease burden, is not necessarily desirable or expected. Even though drugs are an important component of service delivery there are other alternative types of interventions that may be more suitable to address certain types of conditions. Also, some disease areas, such as "mental and behavioral disorders", may be intrinsically more scientifically challenging for innovation than others. However, given the substantial investment in pharmaceutical R\&D and access to drugs, for efficiency and equity reasons, it is important to assess the societal returns on such investments, and in particular, whether current global public/private initiatives are focusing sufficient attention on the priority areas of need.

This analysis has measured the dispersion of drug innovation in terms of disease burden and market size and therefore it has not sought to assess the determinants of differences in innovation across the different disease areas. Moreover, the available data do not allow us to comment on access to drug innovation or its effectiveness when addressing global burden of disease of the population.

Further research might build on this study to develop the accurate measurement of the welfare implications and the value of drug innovation at a global level. Insight from such research is an essential starting point for the design of interventions to better align R\&D innovation with global health needs, and thereby promote equity and efficiency in health systems. 


\section{Acknowledgements}

We would like to thank Owen O'Donnell, Miqdad Asaria, Tommaso Valletti, Rifat Atun, Chris Chapman, Bénédicte Apouey, Fabrice Etilé, and all attendants at the UK Health Economics Study Group, the European Health Economics Conference, Imperial College Business School seminar series, International Health Economics Association conference, and at the workshop Approches Croisées des Inégalités de Santé at the Paris School of Economics, for their comments in previous versions of this analysis.

EB acknowledges financial support available from Fundação para a Ciência e a Tecnologia, Ministério para a Educação e Ciência (FCT), Governo da República Portuguesa, Doctoral Fellowship POPH-QREN-SFRH-BD-69131-2010. FCT had no involvement in the conduct of the research and/or preparation of the article.

Conflicts of interest: Authors have nothing to declare. 


\section{REFERENCES}

Catalá-López, F., García-Altés, A., Álvarez-Martín, E., Gènova-Maleras, R., MorantGinestar, C., \& Parada, A. (2011). Burden of disease and economic evaluation of healthcare interventions: are we investigating what really matters? BMC Health Services Research, 11(1), 75.

Cech, T. R. (2005). Fostering innovation and discovery in biomedical research. JAMA, 294(11), 1390-1393.

Centre for Diseases Control and Prevention. (2011). Neglected Tropical Diseases. Retrieved from http://www.cdc.gov/globalhealth/ntd/diseases/index.html

Clarke, P. M., Gerdtham, U.-G., Johannesson, M., Bingefors, K., \& Smith, L. (2002). On the measurement of relative and absolute income-related health inequality. Social Science \& Medicine, 55(11), 1923-1928.

Drummond, M. F., Wilson, D. A., Kanavos, P., Ubel, P., \& Rovira, J. (2007). Assessing the economic challenges posed by orphan drugs. International Journal of Technology Assessment in Health Care, 23(01), 36-42.

Dukes, M. G. (2002). Accountability of the pharmaceutical industry. The Lancet, 360(9346), 1682-1684.

Erreygers, G. (2009). Correcting the concentration index. Journal of health economics, 28(2), 504-515.

Erreygers, G., \& Van Ourti, T. (2011). Measuring socioeconomic inequality in health, health care and health financing by means of rank-dependent indices: a recipe for good practice. Journal of Health Economics, 30(4), 685-694.

Fauci, A. S., \& Morens, D. M. (2012). The perpetual challenge of infectious diseases. New England Journal of Medicine, 366(5), 454-461.

Friedman, M. (1940). A comparison of alternative tests of significance for the problem of m rankings. The Annals of Mathematical Statistics, 11(1), 86-92 \%@ 0003-4851. 
IMS Health. (2011). IMS Lifecycle User Guide 2011.

IHME (2013). Global Burden of Disease Compare. Available at: http://vizhub.healthdata.org/gbd-compare/ Global Disease Burden study.

Kremer, M., \& Glennerster, R. (2004). Strong medicine: creating incentives for pharmaceutical research on neglected diseases. Princeton, New Jersey: Princeton University Press.

Lopez, A. D., \& Murray, C. (1998). The global burden of disease. Nat Med, 4(11), 12411243.

Marmot, M. (2005). Social Determinants of Health Inequalities. The Lancet, 365(9464), 1099-1104.

Monteiro, C. A., Moura, E. C., Conde, W. L., \& Popkin, B. M. (2004). Socioeconomic status and obesity in adult populations of developing countries: a review. Bulletin of the World Health Organization, 82(12), 940-946.

Murray, C. J., Vos, T., Lozano, R., Naghavi, M., Flaxman, A. D., Michaud, C., . . A Abdalla, S. (2013). Disability-adjusted life years (DALYs) for 291 diseases and injuries in 21 regions, 1990-2010: a systematic analysis for the Global Burden of Disease Study 2010. The Lancet, 380(9859), 2197-2223.

Nathan, C. (2011). Making space for anti-infective drug discovery. Cell host \& microbe, 9(5), 343-348.

Neumann, P. J., Rosen, A. B., Greenberg, D., Olchanski, N. V., Pande, R., Chapman, R. H., . . . Siegel, J. E. (2005). Can we better prioritize resources for cost-utility research? Medical decision making, 25(4), 429-436.

Pammolli, F., Magazzini, L., \& Riccaboni, M. (2011). The productivity crisis in pharmaceutical R\&D. Nature Reviews Drug Discovery, 10(6), 428-438.

Pecoul, B., Chirac, P., Trouiller, P., \& Pinel, J. (1999). Access to essential drugs in poor countries: a lost battle? JAMA, 281(4), 361-367. 
Salomon, J. A., Mathers, C. D., Chatterji, S., Sadana, R., Ustun, T. B., \& Murray, C. J. (2003). Quantifying individual levels of health: definitions, concepts and measurement issues. Health Systems Performance Assessment: Debate, Methods, and Empiricism, 301-318.

Scott Morton, F., \& Kyle, M. (2011). Chapter Twelve - Markets for Pharmaceutical Products1. In T. G. M. Mark V. Pauly \& P. B. Pedro (Eds.), Handbook of Health Economics (Vol. Volume 2, pp. 763-823). Amsterdam: North-Holland/Elsevier.

Trouiller, P., Olliaro, P., Torreele, E., Orbinski, J., Laing, R., \& Ford, N. (2002). Drug development for neglected diseases: a deficient market and a public-health policy failure. The Lancet, 359(9324), 2188-2194.

Van Doorslaer, E., \& Van Ourti, T. (2011). Measuring inequality and inequity in health and health care. In S. Glied \& P. C. Smith (Eds.), Oxford Handbook on Health Economics. Oxford: Oxford University (Vol. 1, pp. 837-869). Oxford: Oxford University Press.

Viergever, R. F., Terry, R. F., \& Karam, G. (2013). Use of data from registered clinical trials to identify gaps in health research and development. Bulletin of the World Health Organization, 91(6), 416-425C.

Wagstaff, A. (2005). The bounds of the concentration index when the variable of interest is binary, with an application to immunization inequality. Health Economics, 14(4), 429-432.

Wagstaff, A., Paci, P., \& Van Doorslaer, E. (1991). On the measurement of inequalities in health. Social Science \& Medicine, 33(5), 545-557.

WHO. (2015). Neglected Tropical Diseases. Retrieved from http://www.who.int/neglected_diseases/diseases/en/

World Bank (2017) World development indicators. Available at: http://data.worldbank.org/data-catalog/world-development-indicators. 
United Nations (2017). Standard country or area codes for statistical use. Available at http://unstats.un.org/unsd/methods/m49/m49regin.htm\#developed. 


\section{TABLES}

Table I: Global R\&D activity and burden of disease

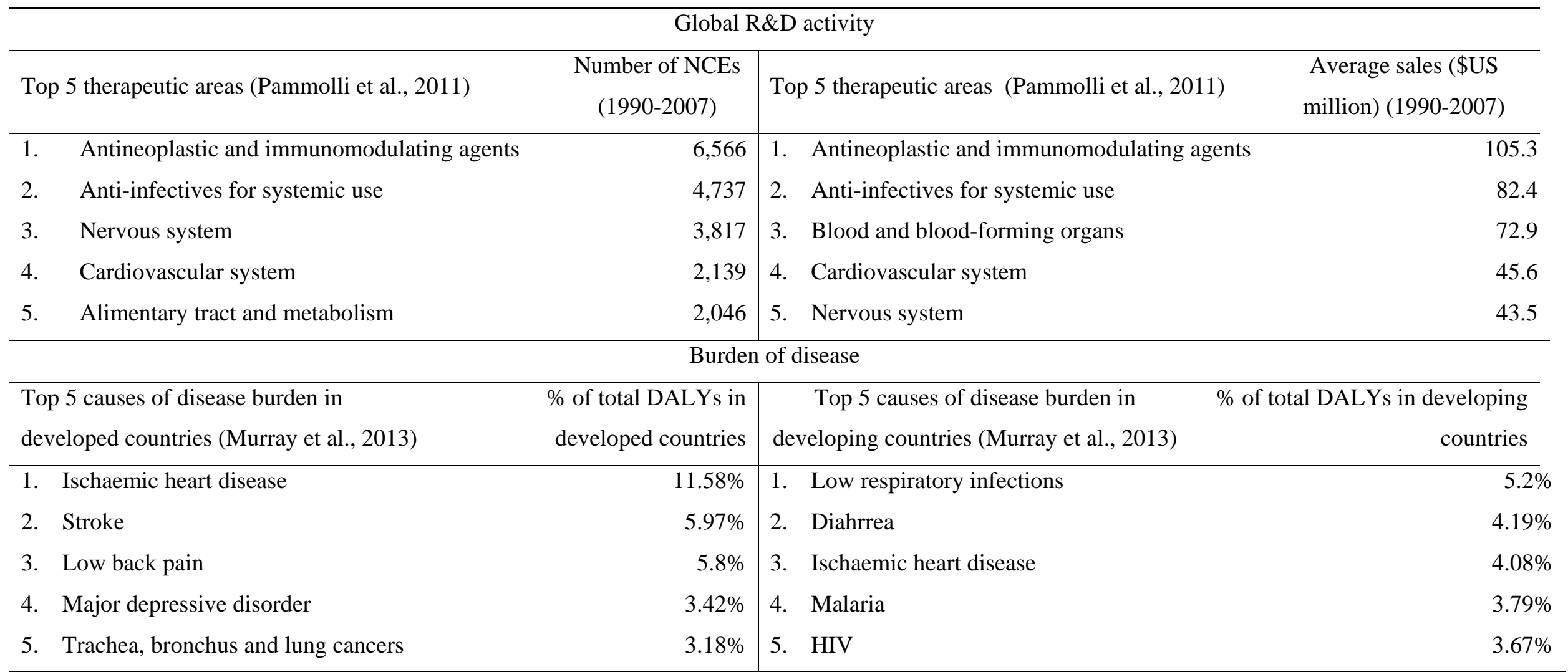

Note: Number of NCEs and average sales for the period between 1990 and 2007 are provided by Pammolli et al. (2011) in the last update about the breakdown of R\&D effort by the pharmaceutical industry at therapeutic level. Top 5 causes of disease burden for developed and developing countries are available online at http://vizhub.healthdata.org/gbd-compare/ Global Disease Burden study (IHME, 2013) and documented in Murray et al. (2013). The 
categorization of developed and developing countries follows the United Nations (United Nations, 2017) classification available at http://unstats.un.org/unsd/methods/m49/m49regin.htm\#developed. 
Table II: List of Neglected Tropical Diseases

\begin{tabular}{ll}
\hline \multicolumn{2}{c}{ Neglected Tropical Diseases } \\
\hline African Sleeping Sickness & Leprosy \\
Buruli ulcer & Lymphatic filariasis \\
Chagas disease & Malaria \\
Cysticercosis & Onchocerciasis \\
Dengue fever & Rabies \\
Dracunculiasis & Schistosomiasis \\
Echinococcosis & Soil-transmitted helminthes \\
Fascioliasis & Trachoma \\
Leishmaniasis & Yaws \\
\hline
\end{tabular}

Source: Centre for Disease Control and Prevention (CDC)

(Centre for Diseases Control and Prevention, 2011) and WHO (WHO, 2015). 
Table III: Descriptive statistics on the global drug innovation across broad disease groups over time

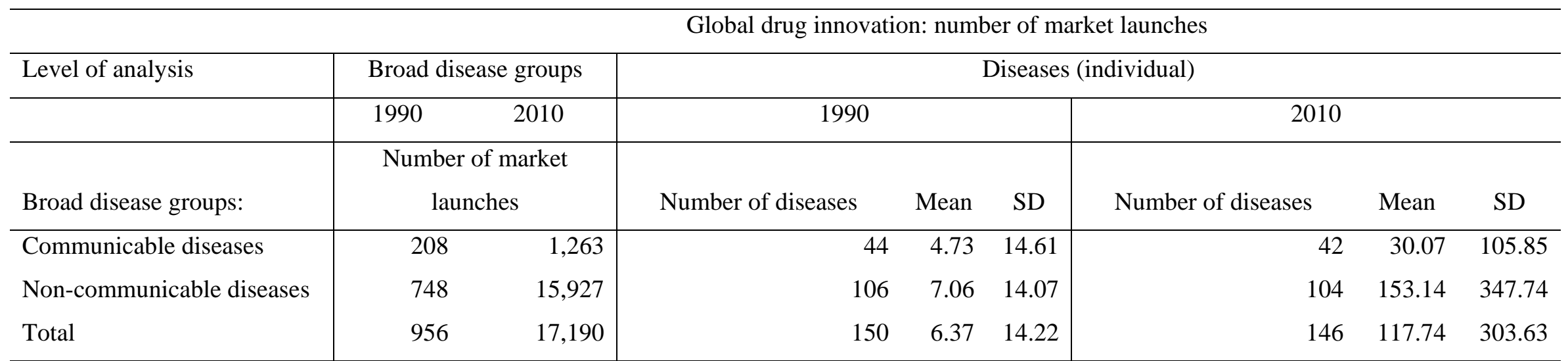

Note: Broad disease groups follow the standard disease taxonomy used by WHO and IHME (Murray et al., 2013).

The broad disease group "Injuries" is excluded from this analysis for not being target for pharmaceutical innovation.

Therefore, excluded from this analysis are the following disease subcategories: transport injuries, unintentional injuries,

self-harm and interpersonal violence, forces of nature, war and legal intervention. 
Table IV: Descriptive statistics on the global drug innovation across disease subcategories over time

Global drug innovation: number of market launches

\begin{tabular}{|c|c|c|c|c|c|c|c|c|}
\hline Level of analysis & Disease subc & ories & & & seases ( & dividual) & & \\
\hline & 1990 & 2010 & & 990 & & & 2010 & \\
\hline Disease subcategories: & Number of $n$ & taunches & $\begin{array}{l}\text { Number of } \\
\text { diseases }\end{array}$ & Mean & SD & $\begin{array}{l}\text { Number of } \\
\text { diseases }\end{array}$ & Mean & SD \\
\hline Cardiovascular and circulatory diseases & 91 & 838 & 4 & 10.11 & 12.02 & $\overline{9}$ & 93.11 & 124.63 \\
\hline Chronic respiratory diseases & 5 & 162 & 4 & 1.25 & 1.5 & 4 & 40.5 & 50.18 \\
\hline Diabetes, urogenital, blood, and endocrine diseases & 90 & 1,721 & 14 & 6.43 & 12.34 & 12 & 143.42 & 345.61 \\
\hline $\begin{array}{l}\text { Diarrhea, lower respiratory infections, meningitis, ar } \\
\text { common infectious diseases }\end{array}$ & 66 & 231 & 13 & 5.08 & 9.15 & 14 & 16.5 & 28.89 \\
\hline Digestive diseases (including cirrhosis of the liver) & 36 & 589 & 7 & 4.86 & 9.49 & 11 & 80.86 & 136.26 \\
\hline HIV/AIDS and tuberculosis & 4 & 46 & 3 & 1.33 & 1.15 & 3 & 15.33 & 9.81 \\
\hline Maternal disorders & 6 & 24 & 6 & 1 & 0 & 6 & 4 & 0 \\
\hline Mental and behavioral disorders & 90 & 1,788 & 15 & 6 & 12.83 & 16 & 111.75 & 258.34 \\
\hline Musculoskeletal disorders & 73 & 1,088 & 3 & 24.33 & 17.21 & 3 & 362.67 & 324.08 \\
\hline Neglected tropical diseases and malaria & 25 & 188 & 9 & 2.78 & 2.05 & 8 & 23.5 & 47.70 \\
\hline Neonatal deficiencies & 0 & 7 & 0 & 0 & 0 & 2 & 3.5 & 3.53 \\
\hline Neoplasms & 285 & 7,670 & 27 & 10.56 & 21.20 & 27 & 284.07 & 570.66 \\
\hline Neurological disorders & 14 & 595 & 7 & 2 & 2.16 & 7 & 85 & 93.85 \\
\hline Nutritional deficiencies & 0 & 0 & 1 & 0 & 0 & 1 & 0 & 0 \\
\hline $\begin{array}{l}\text { Other communicable, maternal, neonatal, and nutriti } \\
\text { (inc. infectious and parasitic diseases) }\end{array}$ & 105 & 760 & 10 & 10.5 & 29.06 & 8 & 95 & 235.77 \\
\hline
\end{tabular}


Other non-communicable diseases: congenital disorders, skin diseases, sense organ diseases, oral conditions

Total

Note: Broad disease groups follow the standard disease taxonomy used by WHO and IHME (Murray et al., 2013). The broad disease group "Injuries" is excluded from this analysis for not being target for pharmaceutical innovation. Therefore, excluded from this analysis are the following disease subcategories: transport injuries

unintentional injuries, self-harm and interpersonal violence, forces of nature, war and legal intervention. 
Table V: Top 4 causes of DALYs and deaths in 1990 and 2010 across regions

\begin{tabular}{|c|c|c|c|}
\hline \multicolumn{4}{|c|}{ Top 4 causes of DALYs and deaths in 1990 and 2010 across regions } \\
\hline \multicolumn{2}{|c|}{ DALYs } & \multicolumn{2}{|c|}{ Deaths } \\
\hline 1990 & 2010 & 1990 & 2010 \\
\hline \multicolumn{4}{|c|}{ Developed Countries } \\
\hline \multicolumn{4}{|c|}{ Central Europe } \\
\hline 1. Ischaemic Heart Disease (IHD) & 1. IHD & 1. IHD & 1. IHD \\
\hline 2. Stroke & 2. Stroke & 2. Stroke & 2. Stroke \\
\hline 3. Low Back Pain & 3. Low back pain & 3. TBLC & 3. TBLC \\
\hline \multicolumn{4}{|l|}{ 4. Trachea, Bronchus and Lung Cancer } \\
\hline (TBLC) & 4. TBLC & 4. Lower respiratory infections (LRI) & 4. Hypertensive heart disease (HHD) \\
\hline \multicolumn{4}{|c|}{ Eastern Europe } \\
\hline 1. IHD & 1. IHD & 1. IHD & 1. IHD \\
\hline 2. Stroke & 2. Stroke & 2. Stroke & 2. Stroke \\
\hline 3. Low Back pain & 3. Low back pain & 3. TBLC & 3. TBLC \\
\hline \multicolumn{4}{|c|}{ 4. Chronic Obstructive Pulmonary } \\
\hline 4. Major Depressive Disorder (MDD) & 4. HIV & Disease (COPD) & 4. HIV \\
\hline \multicolumn{4}{|c|}{ Western Europe } \\
\hline 1. IHD & 1. IHD & 1. IHD & 1. IHD \\
\hline 2. Low back pain & 2. Low back pain & 2. Stroke & 2. Stroke \\
\hline
\end{tabular}




\begin{tabular}{|c|c|c|c|}
\hline 3. Stroke & 3. Stroke & 3. TBLC & 3. TBLC \\
\hline 4. TBLC & 4. MDD & 4. COPD & 4. COPD \\
\hline \multicolumn{4}{|c|}{ High-income Asia Pacific } \\
\hline 1. Stroke & 1. Low back pain & 1. Stroke & 1. Stroke \\
\hline 2. Low back pain & 2. Stroke & 2. IHD & 2. IHD \\
\hline 3. IHD & 3. IHD & 3. LRI & 3. LRI \\
\hline & 4. Other musculos & & \\
\hline 4. Stomach cancer & disorders & 4. Stomach cancer & 4. TBLC \\
\hline \multicolumn{4}{|c|}{ North America } \\
\hline 1. IHD & 1. IHD & 1. IHD & 1. IHD \\
\hline 2. TBLC & 2. COPD & 2. Stroke & 2. Stroke \\
\hline 3. Low Back Pain & 3. Low back pain & 3. TBLC & 3. TBLC \\
\hline & & & 4. Alzheimer diseases and other \\
\hline 4. COPD & 4. TBLC & 4. COPD & dementia \\
\hline \multicolumn{4}{|c|}{ Australasia } \\
\hline 1. IHD & 1. IHD & 1. IHD & 1. IHD \\
\hline 2. Low back pain & 2. Low back pain & 2. Stroke & 2. Stroke \\
\hline 3. Road injuries & 3. COPD & 3. TBLC & 3. TBLC \\
\hline & & & 4. Alzheimer diseases and other \\
\hline 4. Stroke & 4. MDD & 4. COPD & dementia \\
\hline
\end{tabular}

Developing Countries

Sub-Saharan Africa 

1. LRI
1. Malaria
1. LRI
1. Malaria
2. Diahrrea
2. HIV
2. Diahrrea
2. HIV
3. Malaria
3. LRI
3. Malaria
3. LRI
4. Protein energy malnutitrion (PEM)
4. Diahrrea
4. PEM
4. Diahrrea

Latin America and Caribbean

$\begin{array}{llll}\text { 1. Diahrrea } & \text { 1. Disasters } & \text { 1. IHD } & \text { 1. IHD } \\ \text { 2. LRI } & \text { 2. IHD } & \text { 2. Stroke } & \text { 2. Stroke } \\ \text { 3. Preterm birth complications } & \text { 3. Violence } & \text { 3. LRI } & \text { 3. Natural disasters } \\ \text { 4. Interpersonal violence } & \text { 4. Road injuries } & \text { 4. Diahrrea } & \text { 4. LRI }\end{array}$

Southeast Asia, East Asia and Oceania

\begin{tabular}{llll} 
1. LRI & 1.Stroke & 1. Stroke & 1. Stroke \\
2. Stroke & 2.IHD & 2. COPD & 2. IHD \\
3. COPD & 3.COPD & 3. LRI & 3. COPD \\
4. Diahrrea & 4. Road injuries & 4. IHD & 4. TBLC \\
\hline
\end{tabular}

North Africa and Middle East

\begin{tabular}{|c|c|c|c|}
\hline 1. LRI & 1. IHD & 1. IHD & 1. IHD \\
\hline 2. Diahrrea & 2. MDD & 2. Stroke & 2. Stroke \\
\hline 3. Congenital anomalies & 3. Stroke & 3. LRI & 3. LRI \\
\hline 4. IHD & 4. Low back pain & 4. Diahrrea & 4. Road injuries \\
\hline
\end{tabular}

Central Asia

$\begin{array}{llll}\text { 1. LRI } & \text { 1. IHD } & \text { 1. IHD } & \text { 1. IHD } \\ \text { 2. IHD } & \text { 2. LRI } & \text { 2. Stroke } & \text { 2. Stroke } \\ \text { 3. Diahrrea } & \text { 3. Stroke } & \text { 3. LRI } & \text { 3. LRI }\end{array}$


Note: information provided the 2013 Global Burden of Disease study (Murray et al., 2013). The categorization of developed and developing countries follows the United Nations (United Nations, 2017) classification available at http://unstats.un.org/unsd/methods/m49/m49regin.htm\#developed. 
Table VI: Erreygers indices

\begin{tabular}{|c|c|c|c|c|c|}
\hline \multirow[t]{2}{*}{ Broad diseases groups } & \multicolumn{3}{|c|}{ Disease burden } & \multicolumn{2}{|c|}{ Market size } \\
\hline & & DALYs & SE & $\bar{m}$ & SE \\
\hline \multicolumn{6}{|l|}{ Communicable diseases } \\
\hline & 1990 & 0.050 & $(0.038)$ & $0.001 * * *$ & $(0.000)$ \\
\hline & 2010 & 0.068 & $(0.04)$ & 0.008 & $(0.008)$ \\
\hline \multicolumn{6}{|c|}{ Non-communicable diseases } \\
\hline & 1990 & $0.009 * *$ & $(0.030)$ & $0.001 * * *$ & $(0.000)$ \\
\hline & 2010 & 0.017 & $(0.019)$ & $0.039 * *$ & $(0.019)$ \\
\hline \multirow[t]{2}{*}{ Disease subcategories } & & \multicolumn{2}{|c|}{ Disease burden } & \multicolumn{2}{|c|}{ Market size } \\
\hline & & DALYs & SE & $\bar{m}$ & SE \\
\hline
\end{tabular}

TOP4 subcategories of causes of morbidity in the developed countries

Cardiovascular and circulatory diseases

\begin{tabular}{l|ll|ll}
1990 & $0.081^{* * *}$ & $(0.030)$ & $0.062^{* * *}$ & $(0.006)$ \\
2010 & $0.048^{* * *}$ & $(0.048)$ & $0.044^{* * *}$ & $(0.001)$ \\
1990 & & & & \\
2010 & $0.098^{* * *}$ & $(0.016)$ & $0.103 * * *$ & $(0.004)$ \\
1990 & $0.141^{* * *}$ & $(0.022)$ & $0.150^{* * *}$ & $(0.005)$ \\
2010 & $0.267^{* * *}$ & $(0.029)$ & -0.003 & $(0.010)$ \\
1990 & $-0.663^{* * *}$ & $(0.082)$ & 0.011 & $(0.024)$ \\
2010 & -0.019 & $(0.016)$ & $-0.056^{* * *}$ & $(0.005)$ \\
& $-0.115^{* * *}$ & $(0.028)$ & $-0.123^{* * *}$ & $(0.009)$
\end{tabular}

TOP4 subcategories of causes of morbidity in the developing countries

\begin{tabular}{|c|c|c|c|c|c|}
\hline \multicolumn{6}{|c|}{ Diarr+LRI+others } \\
\hline & 1990 & 0.012 & $(0.008)$ & $0.009 * * *$ & $(0.002)$ \\
\hline & 2010 & $0.007 * * *$ & $(0.001)$ & $0.008^{* * *}$ & $(0.000)$ \\
\hline \multicolumn{6}{|c|}{ Neonatal disorders } \\
\hline \multirow[t]{2}{*}{1990 (no obs) } & & missing & & missing & \\
\hline & 2010 & $-0.347 * *$ & $(0.140)$ & $-0.125^{* * *}$ & $(0.028)$ \\
\hline \multicolumn{6}{|c|}{ Cardiovascular and circulatory diseases } \\
\hline & 1990 & $0.081^{* * *}$ & $(0.030)$ & $0.062 * * *$ & $(0.006)$ \\
\hline & 2010 & $0.048 * * *$ & $(0.048)$ & $0.044^{* * *}$ & $(0.001)$ \\
\hline \multicolumn{6}{|c|}{ Nutritional deficiencies (no obs) } \\
\hline
\end{tabular}




\begin{tabular}{ll|ll|ll} 
& 1990 & missing & & missing \\
missing & \\
Neglected tropical diseases + Malaria & 2010 & missing & & & \\
& 1990 & $-0.048^{* * *}$ & $(0.004)$ & $-0.003^{* * *}$ & $(0.001)$ \\
& 2010 & $-0.024^{* * *}$ & $(0.005)$ & $-0.005^{* * *}$ & $(0.001)$ \\
\hline
\end{tabular}

Note: results from Kawkani index and Modified Wagstaff index are qualitatively similar.

The categorization of developed and developing countries follows the United Nations (United Nations, 2017) classification available at http://unstats.un.org/unsd/methods/m49/m49regin.htm\#developed.

Standard Errors in parentheses.** $\mathrm{p}<0.05,{ }^{* * *} \mathrm{p}<0.01$ 
Table VII: Friedman test for diseases ranking: 1990 versus 2010

\section{Friedman test}

\begin{tabular}{|c|c|c|c|}
\hline Disease ranking 1990 versus disease ranking 2010 & Disease burden & Market Size & Innovation \\
\hline \multicolumn{4}{|c|}{ Global } \\
\hline All diseases & $400.338^{* * *}$ & $401.6232 * * *$ & $316.1118^{* * *}$ \\
\hline Communicable diseases & $119.1889 * * *$ & $119.6436^{* * *}$ & $87.6866 * *$ \\
\hline Non-communicable diseases & $282.0664 * * *$ & $249.2209 * * *$ & $236.5554 * * *$ \\
\hline
\end{tabular}

Developed countries

$\begin{array}{llll}\text { All diseases } & 400.64 * * * & 401.7718^{* * *} & 317.3405^{* * *} \\ \text { Communicable diseases } & 119.7481^{* * *} & 119.6467^{* * *} & 92.5469^{* * *} \\ \text { Non-communicable diseases } & 281.1107^{* * *} & 254.1428^{* * *} & 229.2669^{* * *}\end{array}$

Developing countries

\begin{tabular}{lllr} 
All diseases & $400.4457^{* * *}$ & $400.4025^{* * *}$ & $312.9943^{* * *}$ \\
Communicable diseases & $119.0353^{* * *}$ & $119.1214^{* * *}$ & 799.816 \\
Non-communicable diseases & $281.0868^{* * *}$ & $235.6801^{* * *}$ & $230.1946^{* * *}$ \\
\hline
\end{tabular}

Note: The null hypothesis (Friedman) is that the rankings are equal. The categorization of developed and developing countries follows the United Nations (United Nations, 2017) classification available at http://unstats.un.org/unsd/methods/m49/m49regin.htm\#developed. ${ }^{*} \mathrm{p}<0.10 * * \mathrm{p}<0.05$, *** $\mathrm{p}<0.01$.

\section{LEGENDS}

Table I: Global R\&D activity and burden of disease

Table II: List of Neglected Tropical Diseases

Table III: Descriptive statistics on the global drug innovation across broad disease groups over time

Table IV: Descriptive statistics on the global drug innovation across disease subcategories over time

Table V: Top 4 causes of DALYs and deaths in 1990 and 2010 across regions

Table VI: Erreygers indices

Table VII: Friedman test for diseases ranking: 1990 versus 2010 
Figure 1: Inequalities in the drug innovation activity in terms of disease burden in all diseases in 1990 (1a) and 2010 (1b)

Figure 2: Inequalities in the drug innovation activity in terms of disease burden in communicable diseases and non-communicable diseases in 1990 (2a) and 2010 (2b)

Figure 3: Inequality in the drug innovation activity and market size in communicable diseases and noncommunicable diseases in 1990 (3a) and 2010 (3b)

Figure 4: Inequalities in innovation in terms of disease burden in the top four main causes of disease burden in developed countries: cardiovascular and circulatory diseases (4a), neoplasms (4b), musculoskeletal disorders (4c) and mental and behavioural disorders (4d)

Figure 5: Inequalities in innovation in terms of market size in the top four main causes of disease burden in developed countries: cardiovascular and circulatory diseases (5a), neoplasms (5b), musculoskeletal disorders (5c) and mental and behavioural disorders (5d)

Figure 6: Inequalities in innovation activity in terms of disease burden in the top causes of disease burden in developing countries: diarrhea, lower respiratory infections and others (6a), neonatal disorders (6b), and cardiovascular and circulatory disorders (6c).

Figure 7: Inequalities in innovation in terms of market size in the top four main causes of disease burden in developing countries: diarrhea, lower respiratory infections and others (7a), neonatal disorders (7b), and cardiovascular and circulatory disorders (7c).

Figure 8: Inequality in innovation for Neglected Tropical Diseases and Malaria in terms of disease burden (8a) and market size (8b) 


\section{FIGURES}

Figure 1: Inequalities in the drug innovation activity in terms of disease burden in all diseases in 1990 (1a) and 2010 (1b)

(1a)

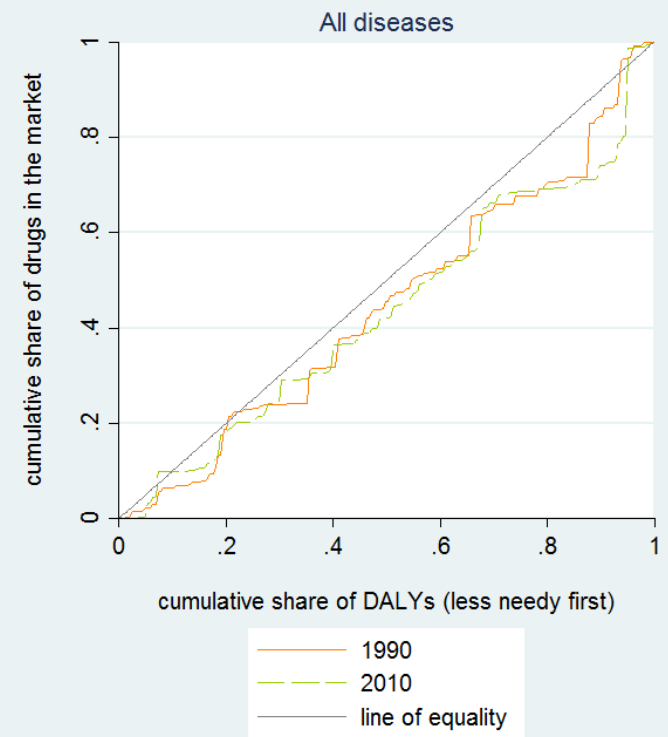

(1b)

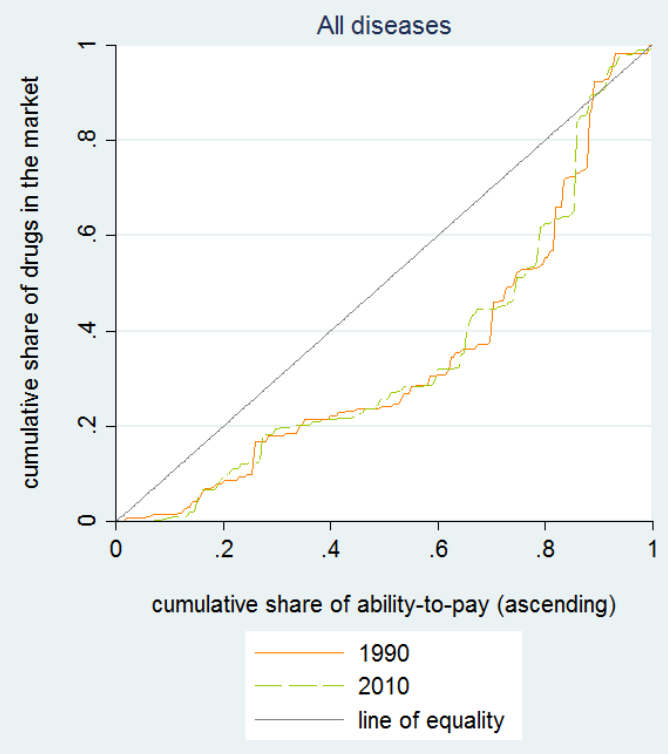


Figure 2: Inequalities in the drug innovation activity in terms of disease burden in communicable diseases and non-communicable diseases in 1990 (2a) and 2010 (2b)

(2a)

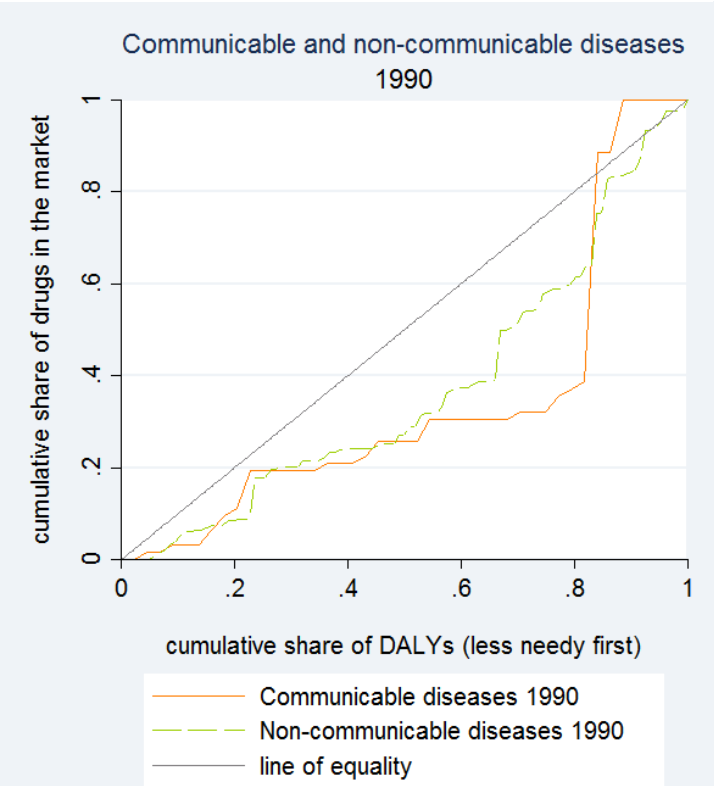

(2b)

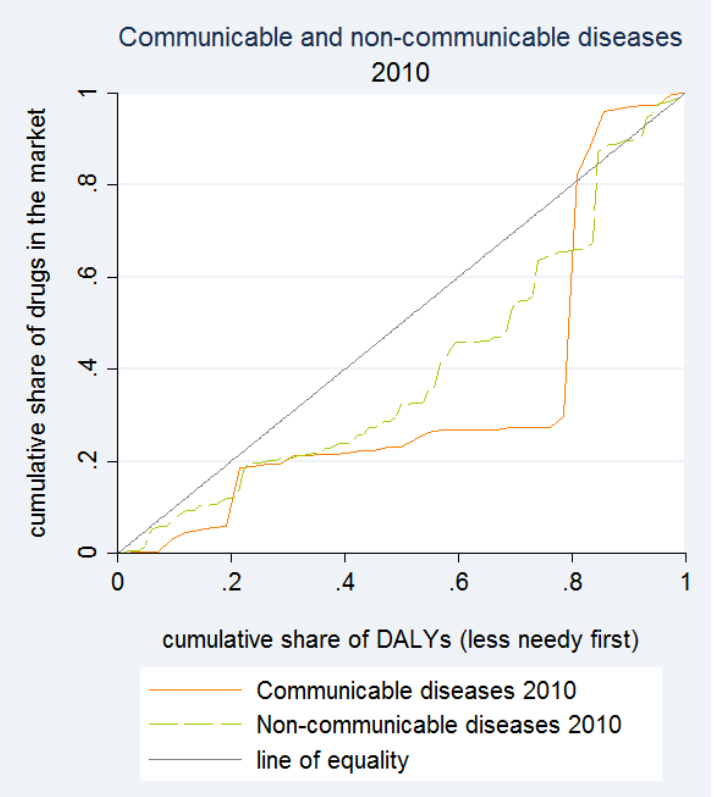


Figure 3: Inequality in the drug innovation activity and market size in communicable diseases and non-communicable diseases in 1990 (3a) and 2010 (3b)

(3a)

(3b)
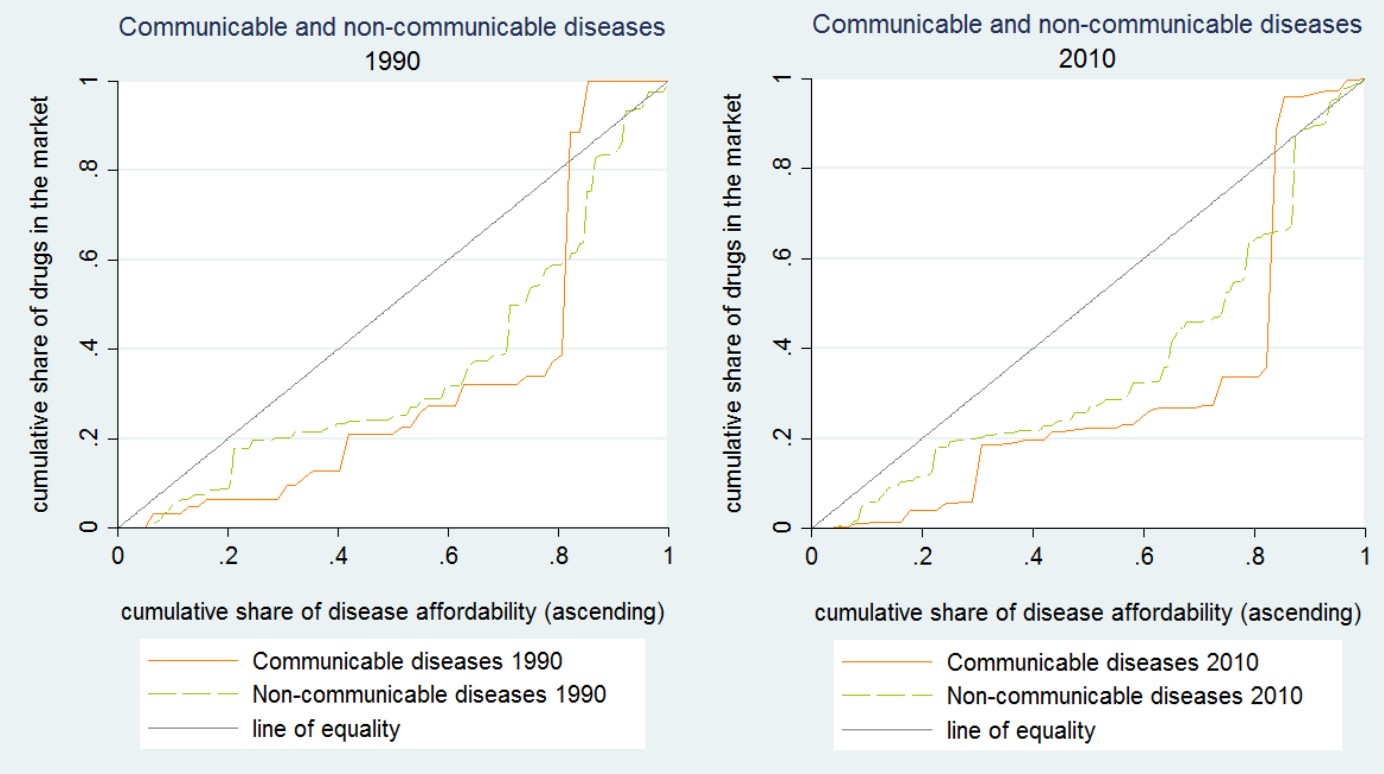
Figure 4: Inequalities in innovation in terms of disease burden in the top four main causes of disease burden in developed countries: cardiovascular and circulatory diseases (4a), neoplasms (4b), musculoskeletal disorders (4c) and mental and behavioural disorders (4d)

(4a)

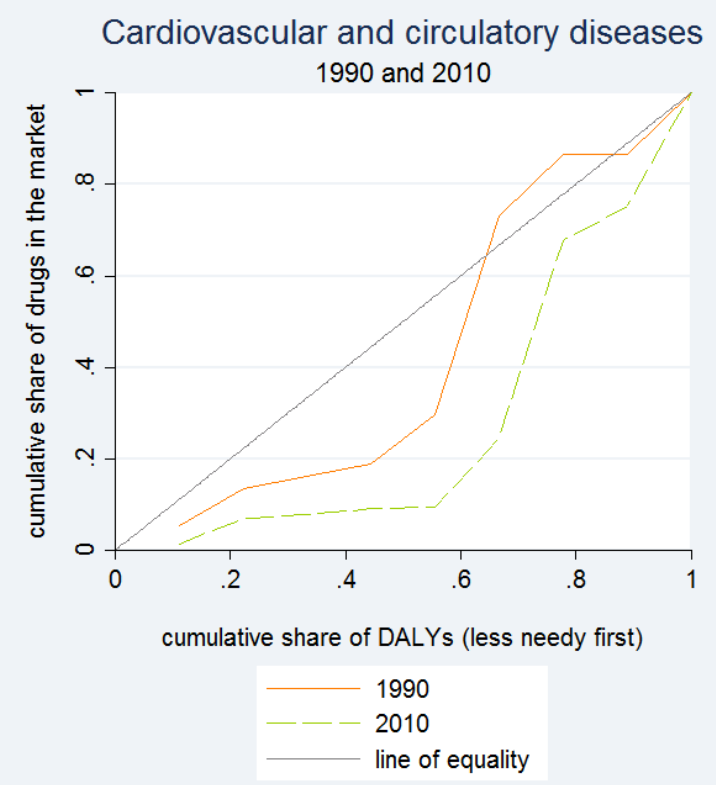

(4c)

(4d)

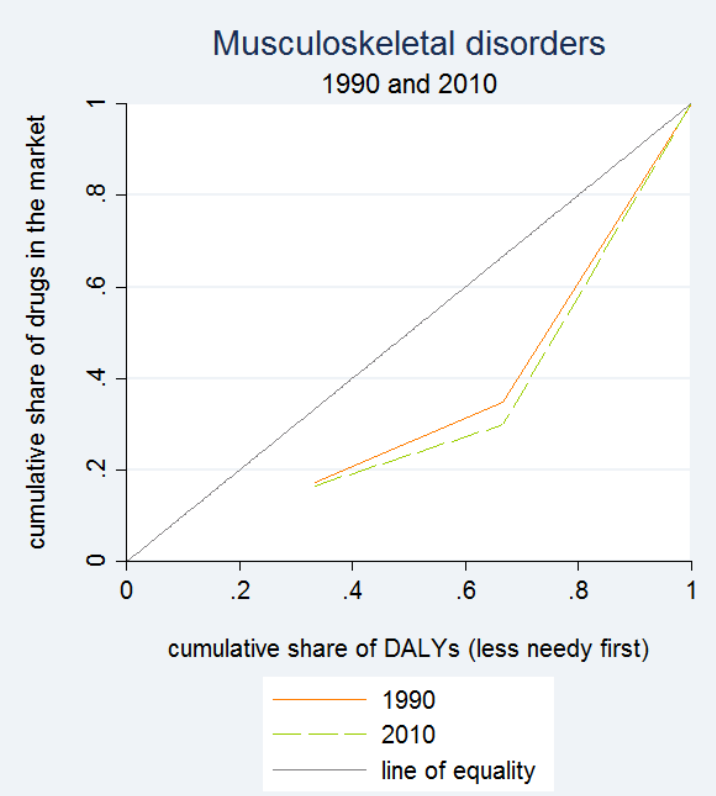

(4b)

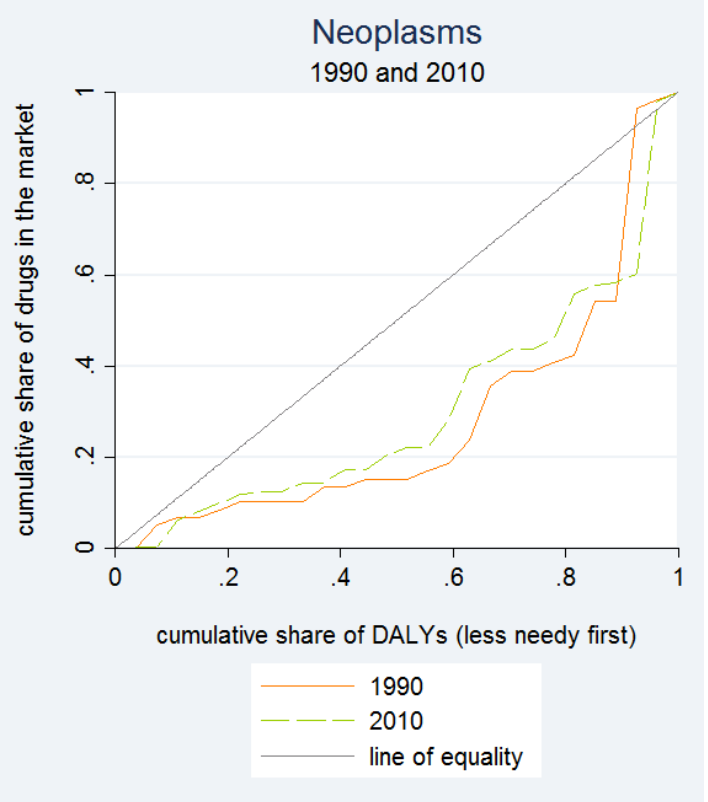

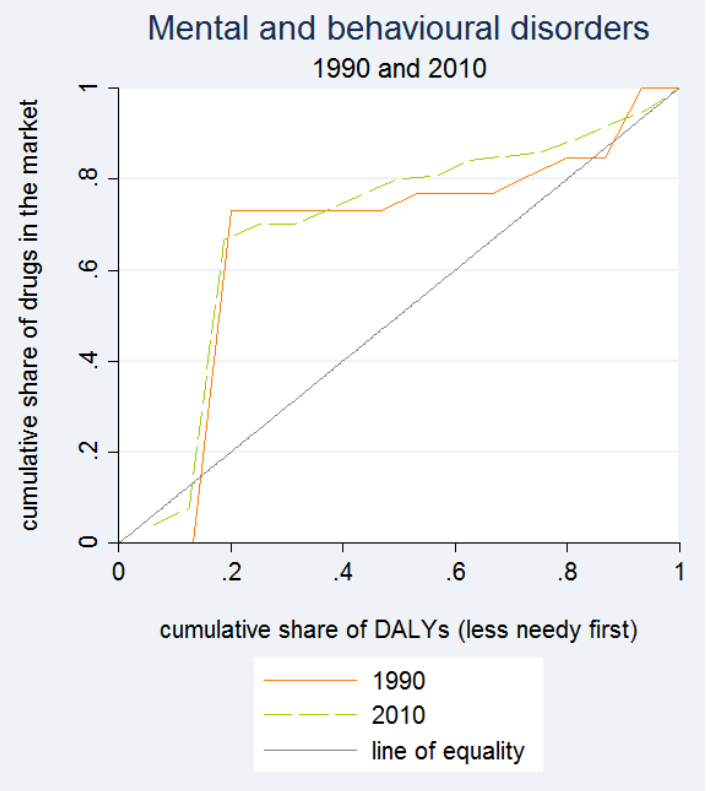

Note: The concentration curves in Figures 4a and 4c start from a point other than the origin, meaning that the first disease in the distribution (i.e. the one that has the lowest disease burden, the lowest on the rank) is responsible for non-zero drugs launched in the market. Curves in Figure 4d show that the first disease in the 
distribution is responsible for zero drugs launched in the market. The categorization of developed and developing countries follows the United Nations (United Nations, 2017) classification available at http://unstats.un.org/unsd/methods/m49/m49regin.htm\#developed. 
Figure 5: Inequalities in innovation in terms of market size in the top four main causes of disease burden in developed countries: cardiovascular and circulatory diseases (5a), neoplasms (5b), musculoskeletal disorders (5c) and mental and behavioural disorders (5d)

(5a)

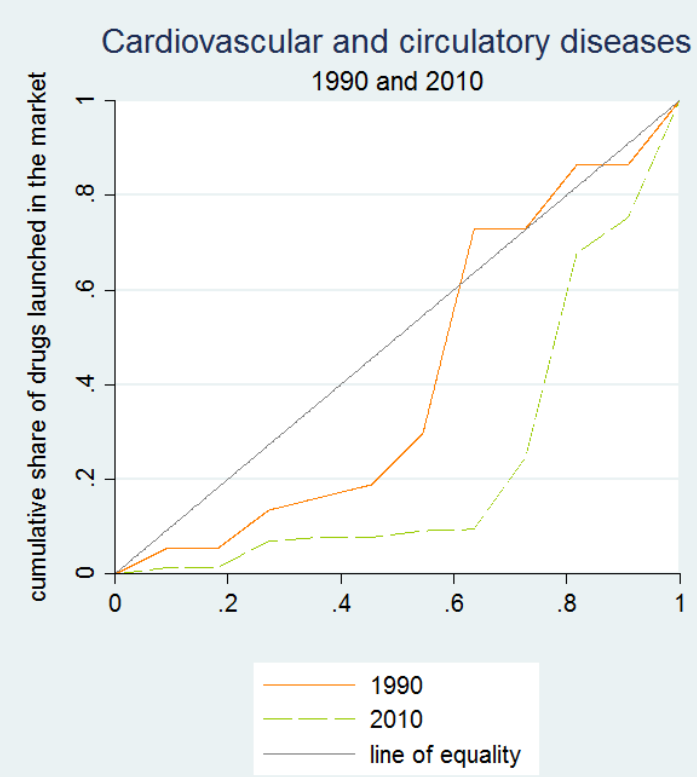

(5b)

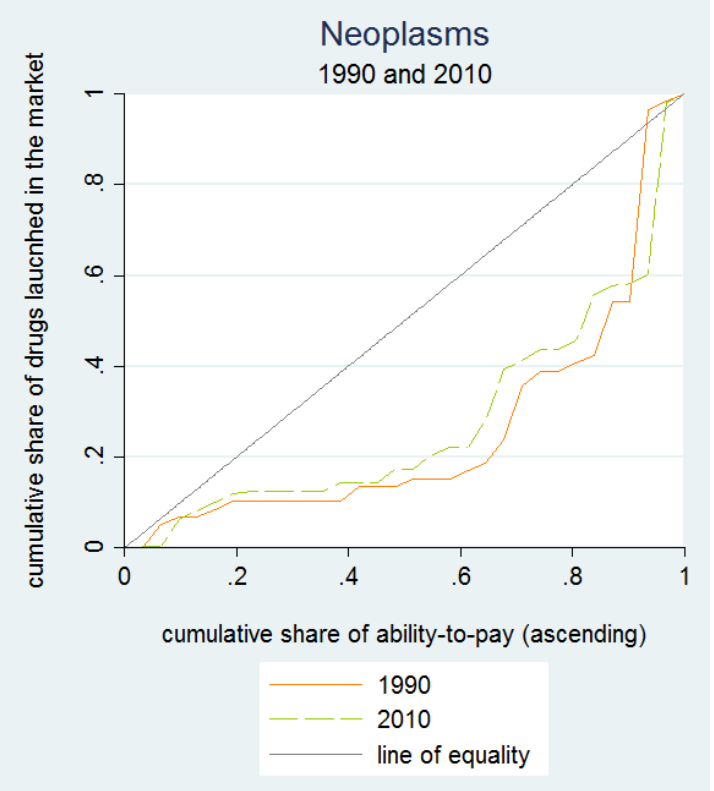

(5c)

(5d)
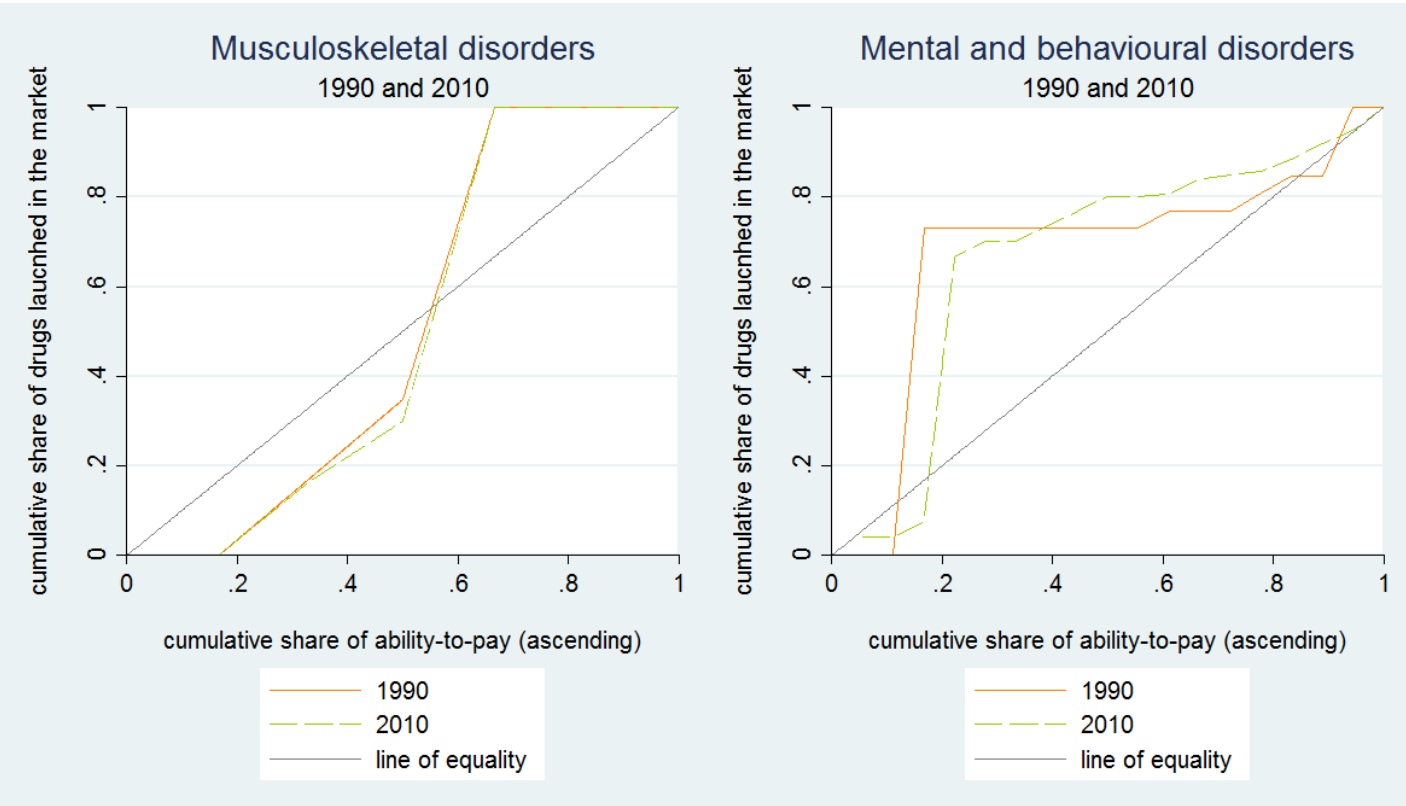

Note: Curves in Figure 5c and 5d start from a point other than the origin, meaning that the first disease in the distribution is responsible for zero drugs launched in the market. The categorization of developed and 
developing countries follows the United Nations (United Nations, 2017) classification available at http://unstats.un.org/unsd/methods/m49/m49regin.htm\#developed. 
Figure 6: Inequalities in innovation activity in terms of disease burden in the top causes of disease burden in developing countries: diarrhea, lower respiratory infections and others (6a), neonatal disorders (6b), and cardiovascular and circulatory disorders (6c).

(6a)

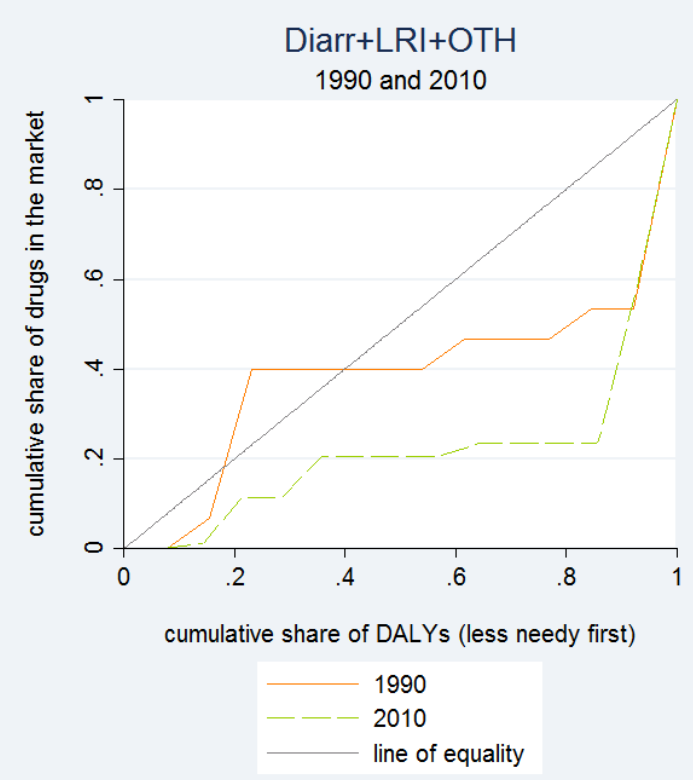

(6b)

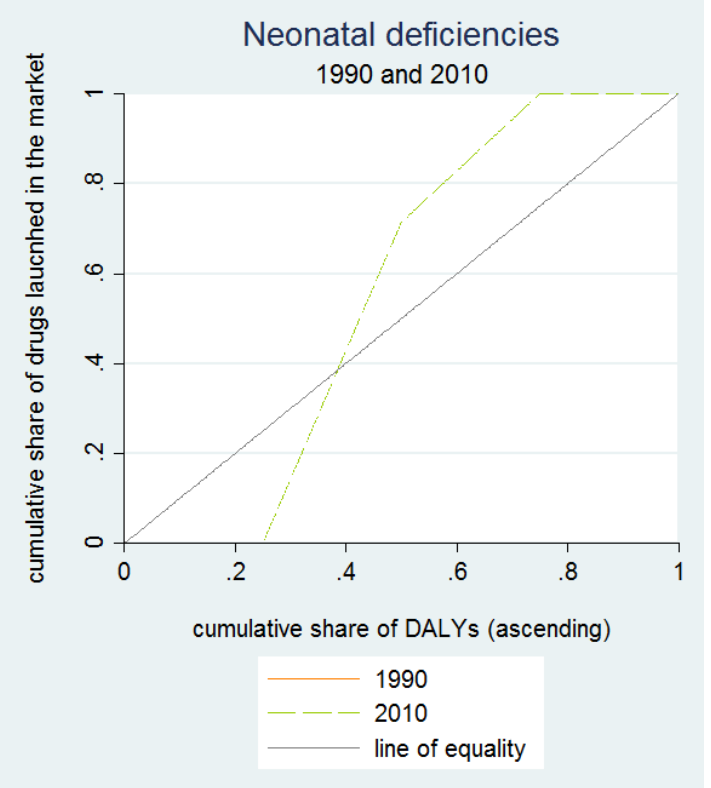

(6c)

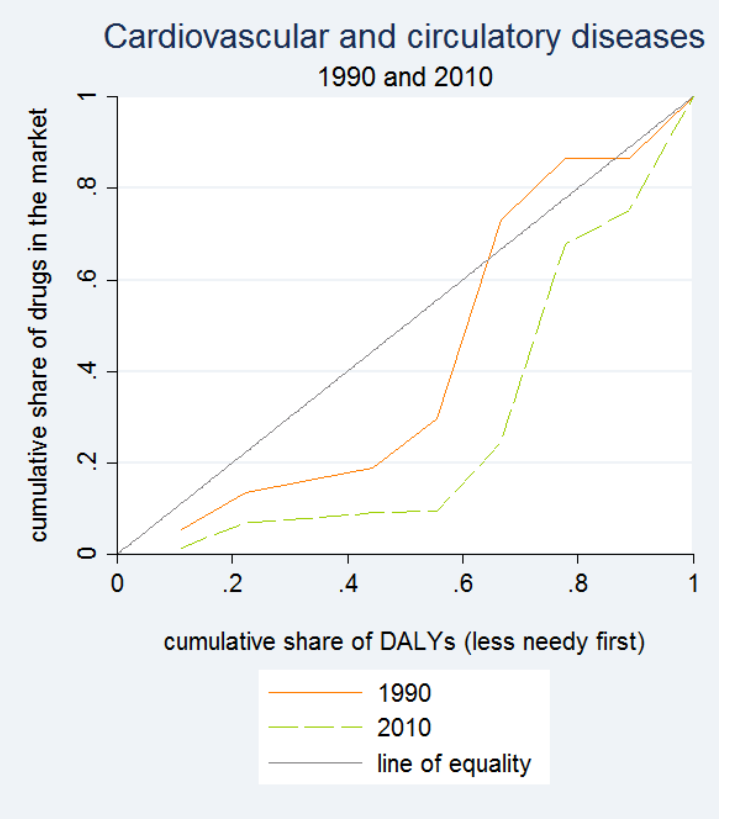

Note: nutritional deficiencies are top ranking in disease burden in developing countries however they do not show any innovation activity. Therefore this disease subcategory is not part of this analysis. There is no innovation activity in year 1990 for any diseases part of neonatal deficiencies. Curves in Figure 6c start from 
a point other than the origin, meaning that the first disease in the distribution (i.e. the one that has the lowest disease burden, the lowest on the rank) is responsible for non-zero drugs launched in the market. Also, Figures $6 \mathrm{a}$ and $6 \mathrm{~b}$ show distributions for which the first disease in the distribution has zero drugs launched in the market. The categorization of developed and developing countries follows the United Nations (United Nations, 2017) classification available at http://unstats.un.org/unsd/methods/m49/m49regin.htm\#developed. 
Figure 7: Inequalities in innovation in terms of market size in the top four main causes of disease burden in developing countries: diarrhea, lower respiratory infections and others (7a), neonatal disorders (7b), and cardiovascular and circulatory disorders (7c).

(7a)
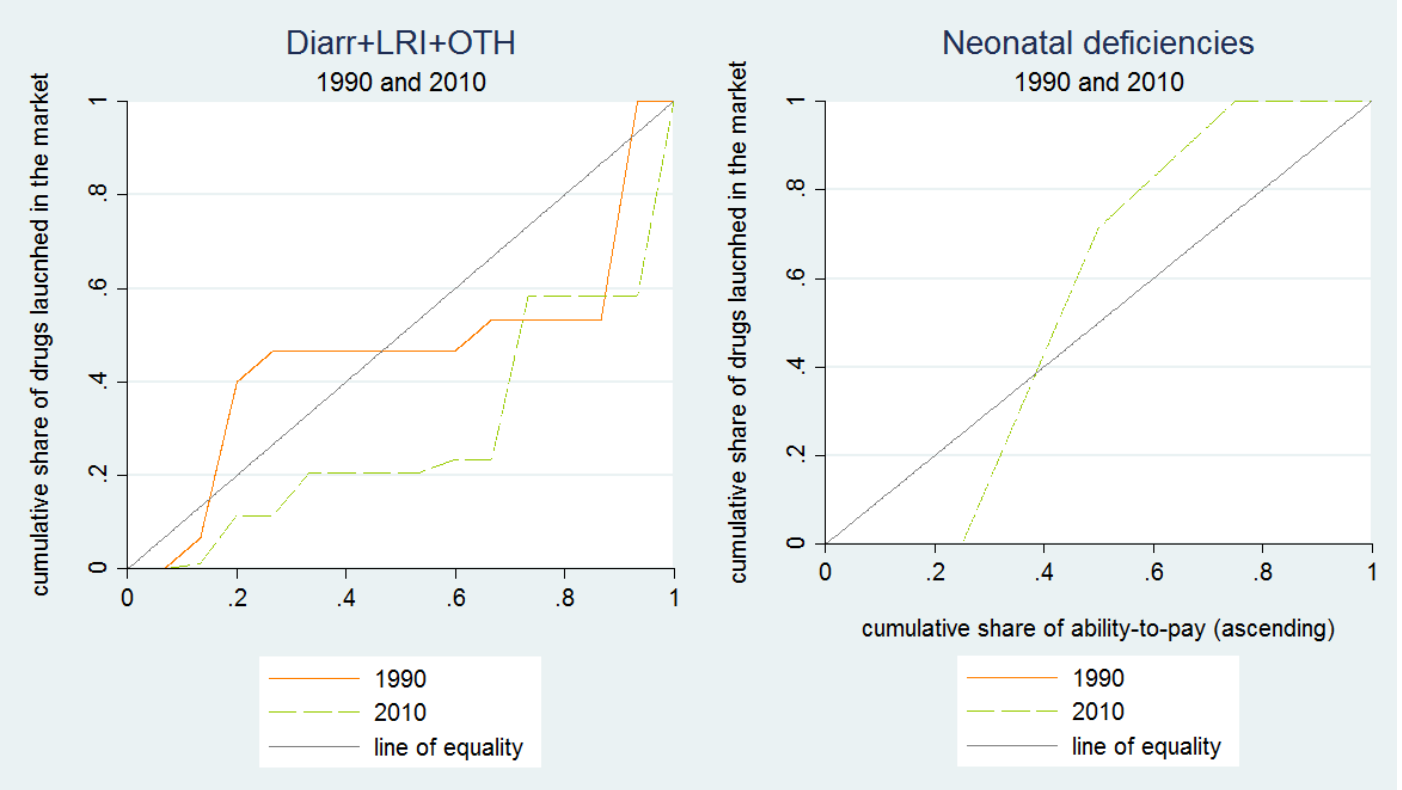

(7c)

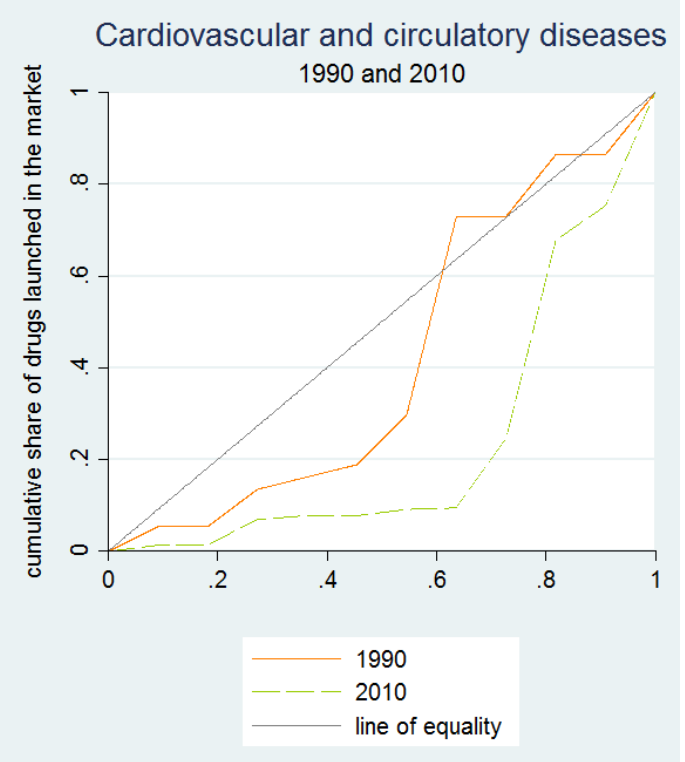

Note: nutritional deficiencies are top ranking in disease burden in developing countries however they do not show any innovation activity. Therefore this disease subcategory is not part of this analysis. There is no innovation activity in year 1990 for any diseases part of neonatal deficiencies. Figures 7a and 7b show curves starting from a point other than the origin, meaning that the first disease in the distribution has zero drugs 
launched in the market. The categorization of developed and developing countries follows the United Nations

(United Nations, 2017) classification available at http://unstats.un.org/unsd/methods/m49/m49regin.htm\#developed. 
Figure 8: Inequality in innovation for Neglected Tropical Diseases and Malaria in terms of disease burden (8a) and market size (8b)

(8a)

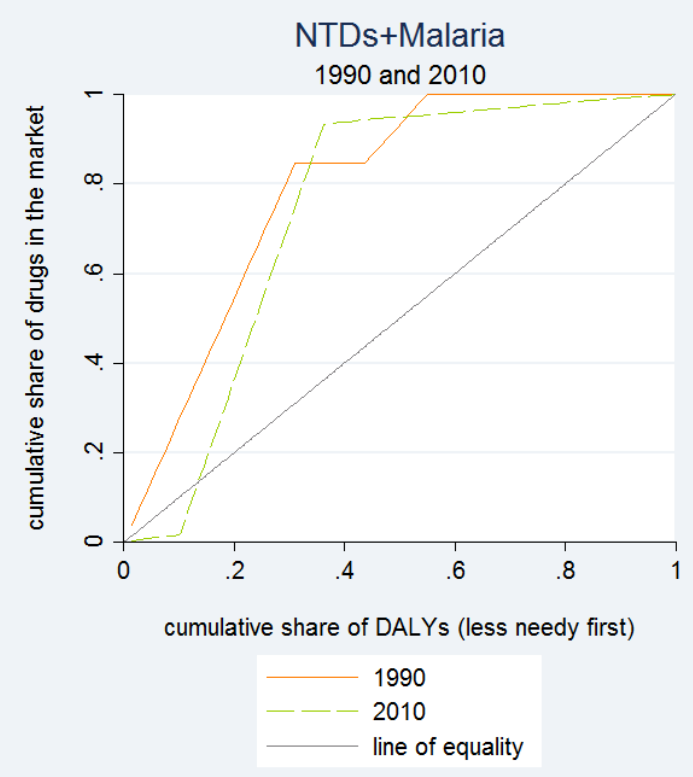

(8b)

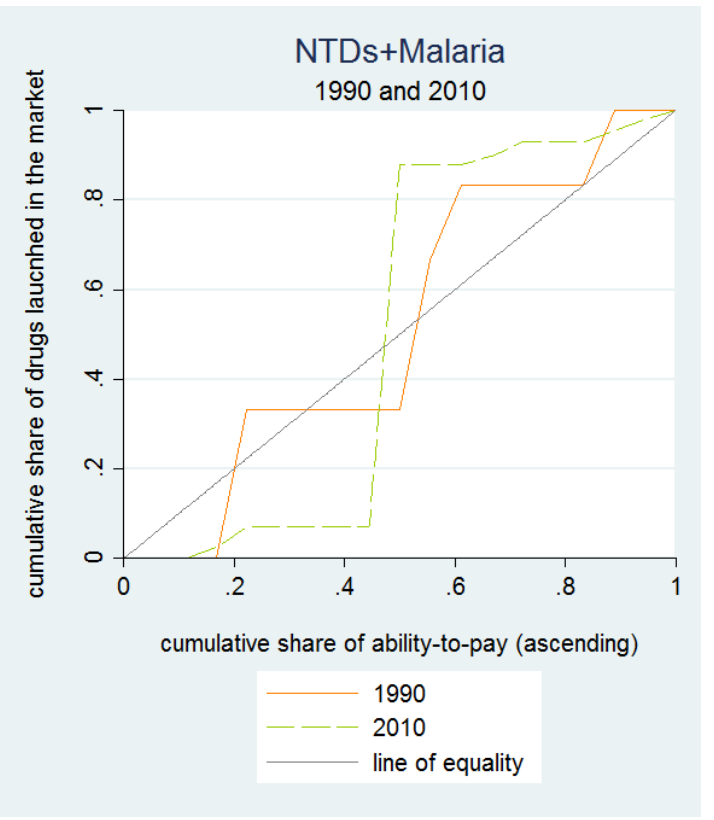

Note: Curves in Figure 8b start from a point other than the origin, meaning that the first disease in the distribution of NTDs (i.e. the one that has the lowest disease burden, the lowest on the rank) has zero drugs launched in the market. 
This paper has been produced by the Department of Management at Imperial College Business School

Copyright (c) the authors 2014

All rights reserved

ISSN: $1744-6783$

Imperial College Business School

Tanaka Building

South Kensington Campus

London SW7 2AZ

United Kingdom

T: +44 (0) 2075895111

F: +44 (o) 2075949184 